\title{
Light Water Reactor Sustainability Program Automated Work Package Prototype: Initial Design, Development, and Evaluation
}

Johanna Oxstrand, Ahmad Al Rashdan, Katya LeBlanc, Aaron Bly, Vivek Agarwal July 2015

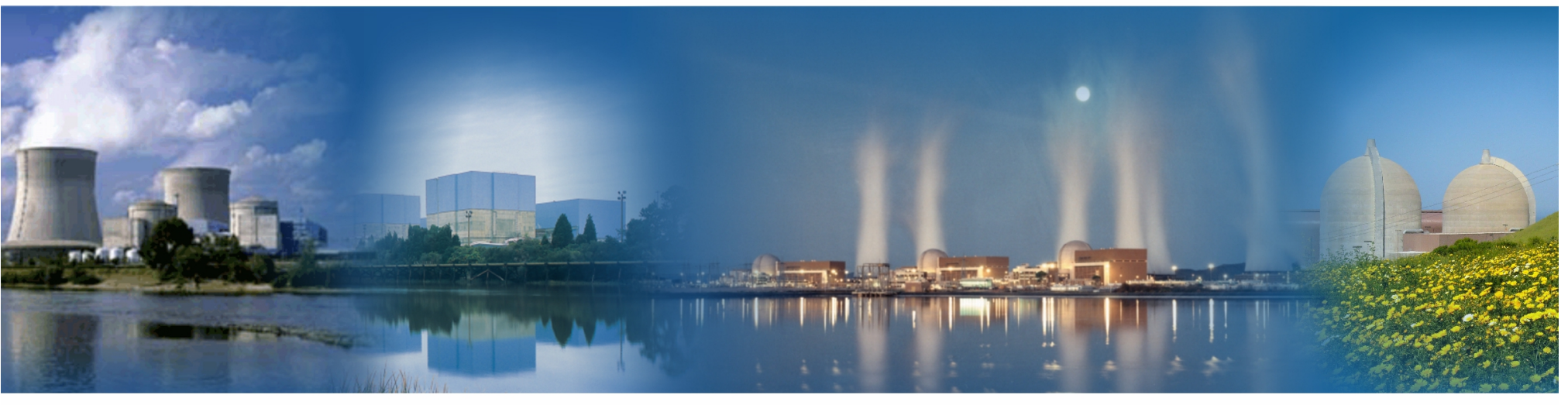




\section{DISCLAIMER}

This information was prepared as an account of work sponsored by an agency of the U.S. Government. Neither the U.S. Government nor any agency thereof, nor any of their employees, makes any warranty, expressed or implied, or assumes any legal liability or responsibility for the accuracy, completeness, or usefulness, of any information, apparatus, product, or process disclosed, or represents that its use would not infringe privately owned rights. References herein to any specific commercial product, process, or service by trade name, trade mark, manufacturer, or otherwise, does not necessarily constitute or imply its endorsement, recommendation, or favoring by the U.S. Government or any agency thereof. The views and opinions of authors expressed herein do not necessarily state or reflect those of the U.S. Government or any agency thereof. 
INL/EXT-15-35825

Revision 0

\title{
Light Water Reactor Sustainability Program Automated Work Package Prototype: Initial Design, Development, and Evaluation
}

\author{
Johanna Oxstrand, \\ Ahmad Al Rashdan, \\ Katya LeBlanc, \\ Aaron Bly, \\ Vivek Agarwal
}

July 2015

\begin{abstract}
Idaho National Laboratory
Light Water Reactor Sustainability Program

Idaho Falls, Idaho 83415
\end{abstract}

http://www.inl.gov/lwrs

Prepared for the

U.S. Department of Energy

Office of Nuclear Energy

Under DOE Idaho Operations Office

Contract DE-AC07-05ID14517 



\section{EXECUTIVE SUMMARY}

The challenges associated with the extension of the service life of existing fleet of nuclear power plants (NPPs) in the United States has driven several directions of research and development as part of the U.S. Department of Energy's (DOE) Light Water Reactor Sustainability Program. One of these directions aims at enabling the development and deployment of new Instrumentation, Information, and Control (II\&C) technologies in existing NPPs. In alignment with this direction, this project aims at improving the efficiency and performance of the execution of maintenance activities, and reducing the probability of the associated human error. To achieve these objectives, it is desired to increase the role of advanced technologies in work packages execution. Instead of the expensive, inefficient, bulky, complex, and prone to errors paper-based work orders, the use of automated work packages (AWPs) is researched and evaluated in this project.

An AWP is a dynamic presentation of the work package designed to guide the user seamlessly through the logical sequence of the process. It allows for a dynamic work flow that depends on the manually and automatically acquired plant and procedure states. It replaces functions where a computer is more efficient and reliable than a human, such as calculations, archived data retrieval, and sequence tracking. An AWP also enables immediate access to resources, such as data sheets and manuals, and a real-time guidance and support to the field operator. A successful implementation of an AWP is strongly dependent on two of its core elements: the computer based procedures (CBPs), and the underlying II\&C technologies. The activities described in this report focused on both of these elements.

The research activities conducted within the human factors scope targeted the improvement of human performance and efficiency related to procedure use and adherence. Two sets of work orders were selected from two participating utilities: Arizona Public Service Palo Verde Nuclear Generating Station (PVNGS) and the Southern Nuclear Company Plant Vogtle. The development of the prototype AWP system used in the field evaluation studies at these plants focused on the design and implementation of several features to enhance the user experience, improve the procedure efficiency, and reduce human errors. The AWP system was used by maintenance technicians at the PVNGS plant during normal operation. The technicians provided feedback about the feasibility of using AWPs in the field and suggestions for further improvements of the user interface. The results of the evaluation are presented in this report. The lessons learned 
from the PVNGS field evaluation study were used to enhance the prototype AWP system developed for Vogtle. The field evaluation study at Vogtle Units 1 and 2 is ongoing, and initial findings are discussed in the report.

The research activities conducted as part of the AWP's underlying II\&C technology focused on the research and development of a communication platform that facilitates a rapid and robust exchange of data between the AWP system, the plants components and other systems of the plant. The platform has the potential to be expandable to a NPP scale and be interoperable with other technologies. This project developed the foundation of the communication platform, taking these initial requirements into consideration. The platform performance was tested on a laboratory-scale flow loop, developed at Idaho State University (ISU) as part of a research on intelligent plant configuration management using wireless sensors (funded by Electric Power Research Institute). The manual valves in the flow loop were fitted with wireless valve indication sensors, which enable real-time valve position status monitoring. In this project, the valves position status was acquired through the developed wireless communication platform into a mobile device. The design, development, and testing of the communication platform highlighted several areas of improvement and concerns to investigate in future research.

The findings of this project encourage further research into the human factors and II\&C perspectives of AWPs. In future work, the results from the CBPs pilot at Vogtle will be evaluated and utilized in the enhancement of future CBPs. In addition, the logic for the existing valve alignment procedure at the ISU flow loop will be mapped to the structure needed for the AWP system. The information from the wireless sensors of the flow loop will be acquired into the AWP, and a study will be performed to evaluate this enhancement. Future II\&C efforts will focus on enhancing the communication framework using wireless communication. Different possible communication strategies will be researched and developed to allow near real-time data acquisition, especially as the size and nature of the wireless communication network changes. Virtual simulations and actual experiments will be performed to evaluate the platform performance characteristics including bandwidth utilization, network scalability, radio frequency interference, and interoperability with different wireless and sensors technologies. Future research efforts will also explore other concealed concerns, solutions and requirements of a large scale implementation of the AWP II\&C infrastructure. 


\section{ACKNOWLEDGEMENTS}

This report was made possible through funding by the U.S. DOE Light Water Reactor Sustainability (LWRS) Program.

The researchers extend their gratitude to Carlos William and Bruce Gordon (PVNGS), James Flowers and Amy Houston (Southern Nuclear Company), and Tim Neal and Teresa Moore (Plant Vogtle) for hosting the two field evaluation studies. The researchers would also want to recognize Jarad Ping and David Delong (PVNGS), and Tracy Quick and Toni Buxton (Plant Vogtle) for supporting the development of the AWP prototype system, and for all their support to plan and conduct the field validation studies.

We also would like to thank Lawrence Beaty from ISU for facilitating the test and demonstration of the data acquisition prototype in the Energy Systems Technology and Education Center. 


\section{CONTENTS}

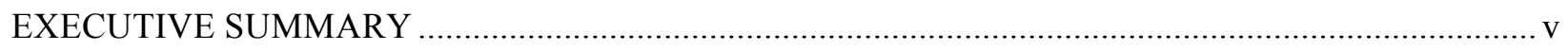

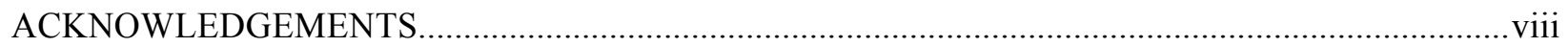

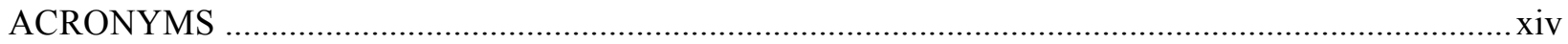

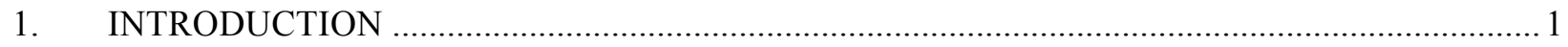

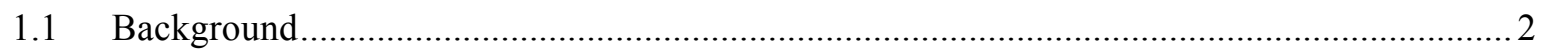

2. HUMAN FACTORS FIELD EVALUATIONS …..............................................................

$2.1 \quad$ Field Evaluation at Palo Verde Nuclear Generating Station ................................................. 4

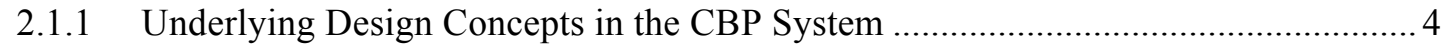

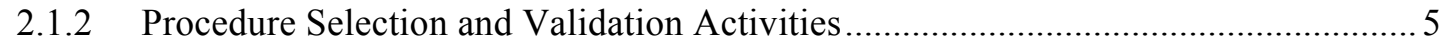

2.1.3 Description of the AWP System Used at PVNGS ............................................... 6

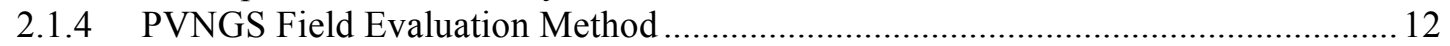

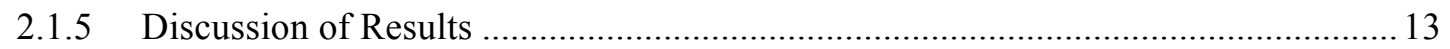

2.2 Field Evaluation at Southern Nuclear Company Plant Vogtle .......................................... 14

2.2.1 Procedure Selection and Validation Activities ......................................................... 14

2.2.2 Description of the AWP System Used at Vogtle.................................................... 15

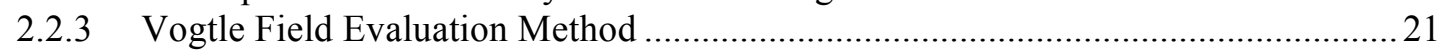

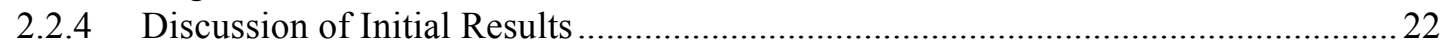

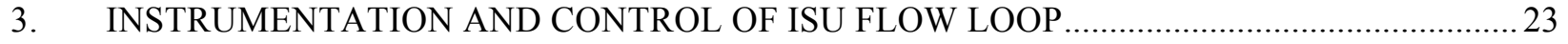

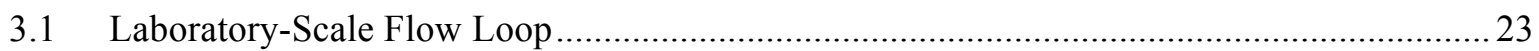

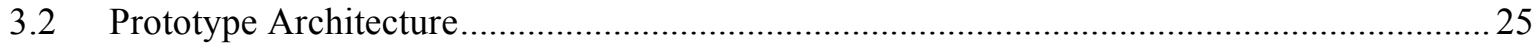

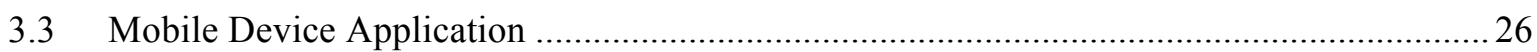

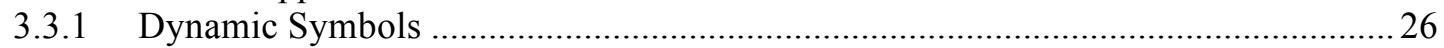

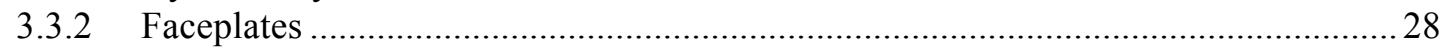

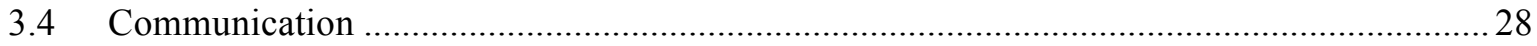

3.4.1 Communication between Field Instrumentation and SQL Database ........................29

3.4.2 Communication between Application on Mobile Device and SQL Database ........... 31

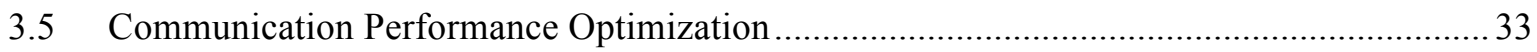

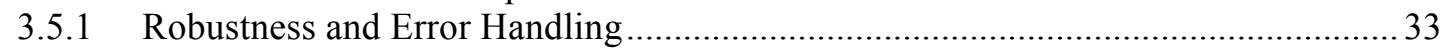

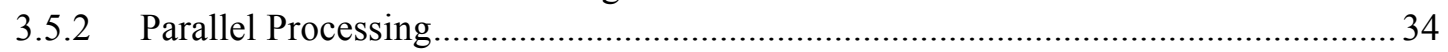

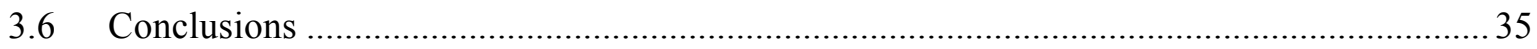

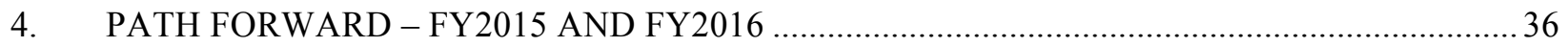

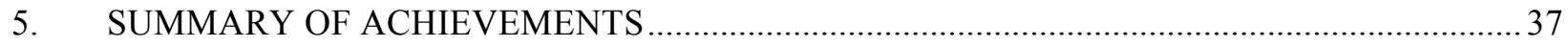

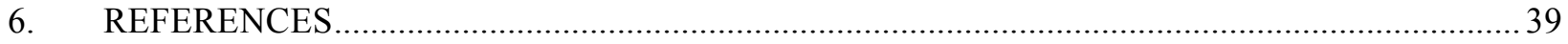




\section{FIGURES}

Figure 1. Analysis of field observation of battery surveillance (Thomas and Lawrie 2015) 3

Figure 2. Screenshots of the basic functionality in the CBP system........................................... 5

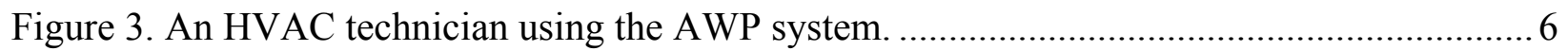

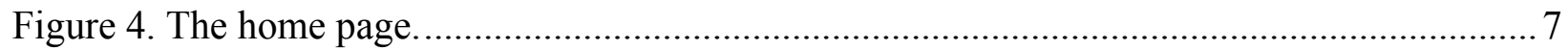

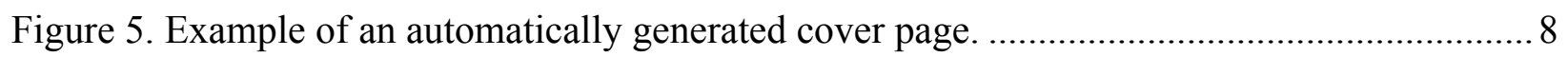

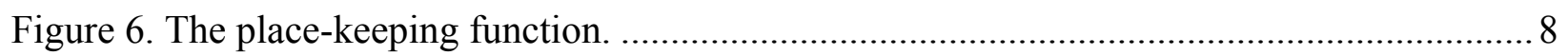

Figure 7. Examples of steps in the HVAC preventive maintenance instruction............................. 9

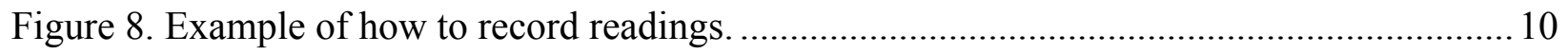

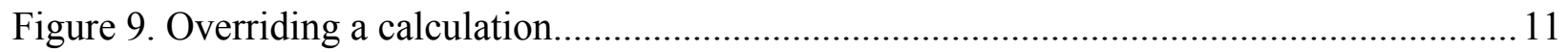

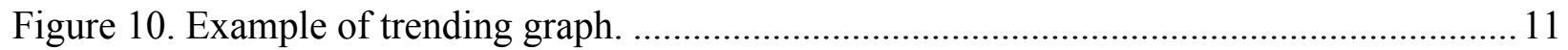

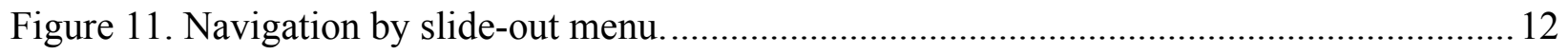

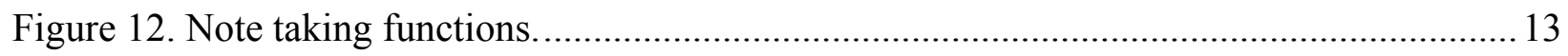

Figure 13. A maintenance technician performing the battery and charger test and

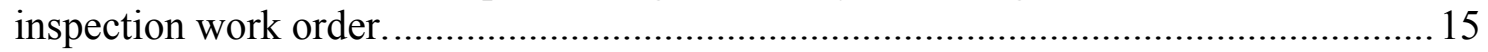

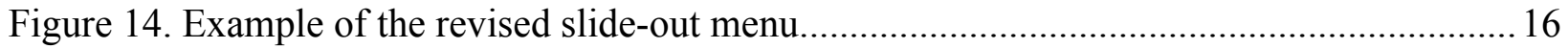

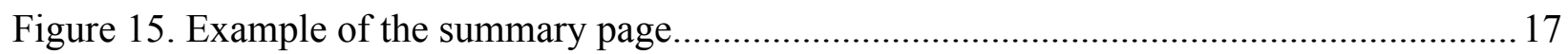

Figure 16. Example of options to preview and start procedure. ………................................... 17

Figure 17. Example of mark-up of pilot cells in the data sheets (cell 42 and 58 are pilot cells here) ............................................................................................................ 18

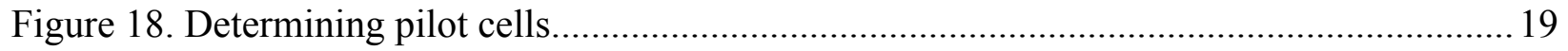

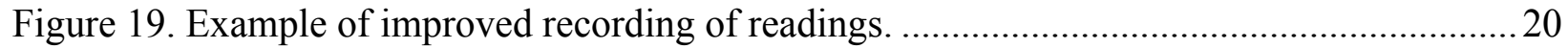

Figure 20. Example of simplified step logic and recorded value out of range. .............................2 21

Figure 21. Example of digital correct component verification. .....................................................22

Figure 22. Picture of the laboratory-scale flow loop (EPRI 2015)........................................... 24

Figure 23. Graphical representation of the laboratory-scale flow loop..........................................25

Figure 24. Wireless network architecture of the prototype...........................................................26

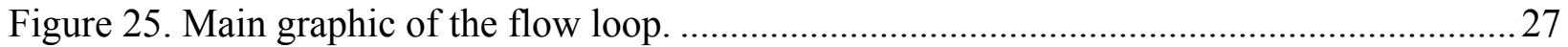

Figure 26. Symbols of valves and their dynamic color of state. ……............................................2 27

Figure 27. Valve's faceplates and calibration faceplate.............................................................. 29

Figure 28. Data format transformation from the field to the tablet................................................. 30

Figure 29. Sequence of steps of the field communication application............................................32 


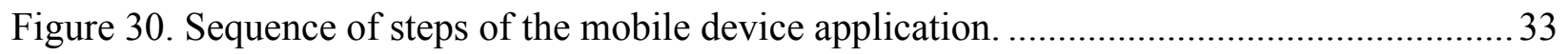

Figure 31. Load sharing wireless network architecture. .....................................................35 


\section{ACRONYMS}

$\begin{array}{ll}\text { ADC } & \text { Analog to Digital Converter } \\ \text { AP } & \text { Access Point } \\ \text { AWP } & \text { Automated Work Package } \\ \text { CBP } & \text { Computer-Based Procedure } \\ \text { CCV } & \text { Correct Component Verification } \\ \text { CPP } & \text { Condensate Polisher Pool } \\ \text { DB } & \text { Database } \\ \text { DOE } & \text { Department of Energy } \\ \text { FY } & \text { Fiscal Year } \\ \text { HVAC } & \text { Heating, Ventilation, and Air Conditioning } \\ \text { IEEE } & \text { Institute of Electrical and Electronics Engineers } \\ \text { II\&C } & \text { Instrumentation, Information, and Control } \\ \text { INL } & \text { Idaho National Laboratory } \\ \text { IP } & \text { Internet Protocol } \\ \text { ISU } & \text { Idaho State University } \\ \text { IT } & \text { Information Technology } \\ \text { LED } & \text { Light Emitting Diode } \\ \text { LWRS } & \text { Light-Water Reactor Sustainability } \\ \text { NPP } & \text { Nuclear Power Plant } \\ \text { OCR } & \text { Optical Character Recognition } \\ \text { PLC } & \text { Programmable Logic Controller } \\ \text { PVNGS } & \text { Public Service Palo Verde Nuclear Generating Station } \\ \text { RF } & \text { Radio Frequency } \\ \text { R\&D } & \text { Research and Development } \\ \text { SQL } & \text { Structured Query Language } \\ \text { TCP } & \text { Transmission Control Protocol } \\ \text { Wi-Fi } & \text { Wireless Fidelity } \\ & \end{array}$




\section{Light Water Reactor Sustainability Program Automated Work Package Prototype: Initial Design, Development, and Evaluation}

\section{INTRODUCTION}

The AWP research effort is a part of the LWRS Program, which is a research and development (R\&D) program sponsored by DOE and performed at INL in close collaboration with the industry's R\&D programs. The LWRS program provides the technical foundations for licensing and managing the long-term, safe, and economical operation of current nuclear power plants. It serves to help the U.S. nuclear industry adopt new technologies and engineering solutions that facilitate the continued safe operation of the plants and extension of the current operating licenses.

Within the LWRS R\&D framework, six areas have been identified that enable capabilities needed for long-term sustainable plant operation. In each of these areas, several pilot projects are planned that enable the development and deployment of new II\&C technologies in existing nuclear power plants. The pilot projects conducted through this program serve as stepping-stones to achieve longer-term outcomes of sustainable II\&C technologies. They are designed to emphasize success in some crucial aspect of plant technology refurbishment and sustainable modernization.

The goal of the AWP project is to demonstrate how to enhance work quality, cost management, and nuclear safety through the use of advanced technology. This will be achieved by combining, integrating, and enhancing the capabilities developed under the mobile technologies and CBP pilot projects, along with developing a real-time (near real-time) plant status capability.

The nuclear power industry is highly proceduralized (i.e., almost all activities that take place at a nuclear power plant are conducted by following procedures). The work planners and/or schedulers plan the scope of work, the task involved, and what procedures should be used for the execution of the task. This information along with other documents, such as pre-job briefs, reference documents, and lists of equipment and tools, are bundled into work packages. These work packages are estimated to be an average of 200 pages and most commonly at least two paper copies will be made of each work package (Thomas and Lawrie 2015).

The paper process has a demonstrated history of ensuring the safety of the industry. Despite the safety record of the current practice there is room for improvement and efficiencies to be gained. The nuclear industry can increase their efficiency and safety by replacing the paper process with an AWP system that improves usability and allows human performance tools to be integrated into the procedure. An AWP system allows for a more dynamic presentation of the work flow to the user. For example, the procedure part of the AWP system can be designed to display only the relevant steps based on operating mode, plant status, and task at hand. In addition, there are aspects where a computer is more efficient and reliable than a human, such as conducting calculations and keeping track of previously recorded values and decisions made.

As suggested by the name, the research activities conducted in the CBP pilot project has a strong focus on how to improve human performance and efficiency related to procedure use and adherence. However, for a system such as a CBP system to be of interest to the nuclear industry, it should encompass more than just procedures for field workers. The system should be able to handle all types of instructions, checklists, procedures, work orders and other documents used in the plant. The grand vision is to have all the different organizations within the plant use the same system and for the system to encompass the whole work package process rather than only focusing on procedures (Oxstrand et al. 2014). 
The success of human performance and efficiency improvement using procedures is strongly dependent on the implemented II\&C technology. It is essential that the technology remains prompt. Slow systems results in negative impact on the overall user experience. The technology should be immune to errors and failures, and report then mitigate failures effects as they occur. Since the nuclear industry has a wide variety of implemented technologies, it is necessary to ensure the technology's interoperability with existing technologies. As a result, a robust, prompt, and flexible technology is essential for a successful implementation of the AWP system. The system architecture of an AWP should be independent of the technology selection. A diverse selection of devices was used to emphasize on cross platforms capabilities.

The II\&C work described in this report is part of the digital architecture for a highly automated plant project of the technical program plan for advanced instrumentation, information, and control systems technologies (Hallbert and Thomas, 2014). To demonstrate the technology developed in this work, a prototype for plant components status acquisition to a mobile device was designed and developed at INL then tested and demonstrated on a laboratory-scale flow loop at ISU. The flow loop was equipped with an intelligent plant configuration management using wireless sensors as part of another project (EPRI, 2015).

This report addresses the DOE Milestone M2LW-15IN0603112: Describe the outcomes of field evaluations/demonstrations of the AWP prototype system and plant surveillance and communication framework requirements at host utilities. There are a couple of different parts needed to adequately address the milestone. First, a brief background to the need for AWP research will be provided, then two human factors field evaluation studies will be described (Section 2). These studies focus on the user experience of conducting a task (in this case a preventive maintenance and a surveillance test) while using an AWP system. The other main part of the report is Section 3, which describes an II\&C effort to provide real time status updates to the technician by wireless transfer of equipment indications and a dynamic user interface.

\subsection{Background}

The LWRS II\&C pathway has developed a standard methodology for determining the impact of new technologies in order to assist nuclear power plants in building sound business cases to support the implementation of the advanced technologies developed in the LWRS program. As a part of the business case effort, Thomas and Lawrie (2015) developed a business case for mobile work packages. Some of the work orders reviewed to build the business case are very similar to the work orders used for field evaluation studies in the AWP project. For example, one of the work orders reviewed is a battery surveillance test, which has a strong resemblance of the battery and charger test and inspection used for the Vogtle 1 and 2 field evaluation study (described in detail later in the report). As shown in Figure 1, Thomas and Lawrie estimated a potential 14\% efficiency gain and more than 3.5 hours of time saving when using a mobile work package compared to the current paper-based work process for the battery surveillance test.

Thomas and Lawrie also concluded that modest efficiency gains can be realized during execution of the procedure. However, the largest efficiency gains will occur as a result of efficiencies in pre-job preparation and post-job closing activities, as well as the reduction of wait times and recovery from work stoppages and other events that introduce interruptions to the execution of work.

Based on the observations conducted to support the development of the business case it was concluded that most of the human error reduction opportunities are derived from mobile work packages and CBP features that support field work execution. This conclusion shows the importance and value of the human factors research conducted in both the LWRS program and in the AWP project in particular. The research activities in the AWP project aim to evaluate the functionalities and aspects of the complete work package process (i.e., the full process from initiating work request, planning, execution, and archiving) (Oxstrand and Le Blanc 2015; Oxstrand et al 2015; Agarwal et al., 2014.) 


\begin{tabular}{|c|c|c|c|c|c|c|c|}
\hline $\begin{array}{l}\text { Time } \\
\text { Start }\end{array}$ & $\begin{array}{l}\text { Time } \\
\text { End }\end{array}$ & Procedure Step & Description of Activity & $\begin{array}{l}\text { Observed } \\
\text { Duration } \\
\text { (h:mm:ss) }\end{array}$ & $\begin{array}{l}\text { Estimated } \\
\text { Time } \\
\text { Savings } \\
\text { (h:mm:ss) }\end{array}$ & $\begin{array}{c}\text { Estimated } \\
\text { Efficiency } \\
\text { (\%) }\end{array}$ & Comments \\
\hline $7: 30$ & $8: 15$ & & Pull M\&TE & $0: 45: 00$ & $0: 05: 00$ & $89 \%$ & \\
\hline $8: 15$ & $9: 02$ & $1.0,2.0,3.1-3.5$ & Task Cover Sheet / Pre-Job Brief & $0: 47: 00$ & $0: 10: 00$ & $79 \%$ & \\
\hline 9:02 & 9:06 & & Travel (2) to Ops & $0: 04: 00$ & & $100 \%$ & \\
\hline 9:06 & $9: 15$ & $3.6-3.10$ & Sign out job (Ops) & 0:09:00 & 0:04:00 & $56 \%$ & \\
\hline $9: 15$ & $9: 24$ & 4.1 & Inspection and Maintenance & $0: 09: 00$ & & $100 \%$ & \\
\hline $9: 24$ & $9: 26$ & 4.2 & Battery terminal voltage measurement & $0: 02: 00$ & & $100 \%$ & \\
\hline $9: 26$ & $9: 31$ & 4.3 & $\begin{array}{l}\text { leave battery room, go to charger room, verify } \\
\text { tags - go to } 4.3 .3 \text {. }\end{array}$ & $0: 05: 00$ & 0:01:00 & $80 \%$ & \\
\hline $9: 31$ & $9: 33$ & $4.3 .3 .1-2$ & Verify readings - hold work & $0: 02: 00$ & & $100 \%$ & \\
\hline $9: 33$ & $10: 22$ & & Approve work-around & $0: 49: 00$ & $0: 02: 27$ & $95 \%$ & $\begin{array}{l}\text { Uncommon issue, but take } 5 \% \\
\text { savings for avoided interuptions }\end{array}$ \\
\hline $10: 25$ & $10: 28$ & 4.5 & Data sheet 1 Float Current & 0:03:00 & $0: 00: 30$ & $83 \%$ & \\
\hline $10: 36$ & $10: 44$ & 4.6 & Data sheet 2 Cell float Voltage & $0: 08: 00$ & $0: 00: 30$ & $94 \%$ & \\
\hline $10: 44$ & $10: 48$ & 4.7 & Data sheet 2 Electrolyte Level & 0:04:00 & 0:00:30 & $88 \%$ & \\
\hline $10: 48$ & $10: 55$ & 4.8 & Data sheet 2 Specific Gravity (4 pilot cells) & $0: 07: 00$ & $0: 00: 30$ & $93 \%$ & \\
\hline $10: 55$ & $11: 02$ & 4.10 & Skip 4.9-Visual cell and rack inspection & $0: 07: 00$ & & $100 \%$ & \\
\hline $11: 02$ & $11: 15$ & & Close out work / cleanup / Return to shop & $0: 13: 00$ & & $100 \%$ & \\
\hline $11: 15$ & $11: 45$ & 4.15 & $\begin{array}{l}\text { Post job (estimated) - signout, package } \\
\text { sorting, approval, records, etc. }\end{array}$ & $0: 30: 00$ & $0: 10: 00$ & $67 \%$ & $\begin{array}{l}\text { May include work done by } \\
\text { Admins }\end{array}$ \\
\hline $7: 30$ & 11:45 & & & $4: 15: 00$ & $0: 34: 27$ & $86 \%$ & \\
\hline
\end{tabular}

Figure 1. Analysis of field observation of battery surveillance (Thomas and Lawrie 2015).

The other perspective of the research activities in the AWP project aims to evaluate the technologies necessary to implement the AWP in a nuclear power plant. This includes the research and development of instrumentation and communication infrastructures to facilitate the communication of the plant's components status to the AWP system, and to facilitate the communication of the AWP system elements.

Relatively modern plants, such as Vogtle 1 and 2, have the instrumentation infrastructure for implementing such advanced solutions. Other older reactors lack such infrastructure as and require alternative novel approaches. An earlier effort (EPRI 2015) demonstrated an evident potential for instrumenting manually operated valve's that are not originally equipped with status indications. Research in technologies and methods to instrument other currently non-instrumented parts of nuclear power plants are expected to continue.

Since several nuclear power plants lack the cabling infrastructure that is needed to link these instruments to a monitoring station, a wireless network is the most feasible solution. The use of wireless communication has an economical and applicability advantage over wired communication. Nuclear power plants already have various extends of wireless networks. Plants like South Texas Project had 440 access points in 2014 (EPRI 2014b). Others have no wireless network at all. While the installed networks were not originally intended for wireless instrumentation, they reveal the feasibility of a wireless network implementation.

Despite the economical and feasibility advantages of wireless communication, concerns have been raised regarding their performance in a highly demanding environment. Topics such as bandwidth, robustness, response time, interference, and security are some of the common concerns associated with wireless communication (Hashemian, et. al. 2011). These concerns along with other requirements are listed in an earlier report of this project (Agarwal, Oxstrand, and Le Blanc 2014). This work targets the requirements of a platform that is prompt, robust and interoperable with any technology. 


\section{HUMAN FACTORS FIELD EVALUATIONS}

Previous research on work instructions for field workers conducted in the LWRS program focused on procedures for field operators. The AWP research team aims to expand that research to include work orders, associated processes, and instructions. Two human factors research activities have been conducted in the AWP project, one field evaluation study at PVGNS and one at Plant Vogtle 1 and 2. The purpose of these activities was to test a prototype AWP system in actual plants during normal operation and to gather feedback from maintenance technicians about their experience with the system. The technicians used the AWP system developed by the research team to conduct preventive maintenance work orders and surveillance tests. The goal was to evaluate the new functionality added to the system and the overall user experience. The researchers investigate the feasibility of applying the design concepts developed in the CBP project to instructions found in work packages, (i.e., target to maintenance organizations).

In addition, the field evaluation studies are an attempt to make the research effort more accessible and relatable to the nuclear industry. It is assumed that many questions about hurdles related to and the deployment of an AWP system will be easier addressed when both senior management and the work force get to use the INL prototype for actual work in the plant. These efforts pose a non-binding commitment to the utilities, which a similar activity provided by a vendor might do.

\subsection{Field Evaluation at Palo Verde Nuclear Generating Station}

The first field evaluation study conducted by the researchers was hosted by the Arizona Public Service PVNGS. The AWP system developed for the field evaluation study built on a CBP system developed in a parallel project in the LWRS program.

This section briefly describes the basic functionalities and design concepts of the CBP system and the revisions made to encompass the needs of the AWP project. A summary of the field evaluation study at PVGNS is also provided.

\subsubsection{Underlying Design Concepts in the CBP System}

To better understand the additions made to the previously developed system for CBPs one should be aware of a couple of basic functions incorporated in the CBP system that does not exist in current paper-based procedures. The dynamic nature of a computer-based system compared to the static nature of paper enables these functions. For more details on the design concepts behind the CBP system, see Oxstrand et al., 2014. The most important design concepts in the CBP system are; automatic place-keeping, simplified step logic, and digital correct component verification.

Automatic place-keeping. The CBP system highlights the active step (i.e., the step being conducted). Other steps are shown, but the user can only take actions related to the active step. The CBP system decides what step is next in line to be executed and what step(s) to mark as Not Applicable based on the actions taken in previous steps, user inputs, and plant status. The automatic place-keeping makes it easy for the field worker to stay on the specified path. This built-in procedural adherence has proven to reduce the amount and severity of human errors. The step to take action is clearly marked with a blue border, see Figure 2.

Simplified step logic. A conditional step in a procedure is a step that is based on plant conditions or a combination of conditions to be satisfied prior to the performance of an action. The CBP removes complexity from step descriptions by presenting conditional statements as simple questions, paired with condition-specific instructions. For example, statements such as "IF this is the initial run, THEN perform the following..." are presented as "Is this the initial run, Yes or No?" as shown in Figure 2. Depending on the answer, the procedure will take the field worker to the step with the next required action.

Digital correct component verification. Before taking an action on a component or piece of equipment, the field worker is required to verify that he/she is at the right component. This is called 
correct component verification (CCV). Currently, this is carried out by looking at the procedure and reading the component identification aloud. Then, the field worker will touch the component's label and read the component identification aloud. If there is a match, the correct component has been verified. However, incidents where the field worker manipulates the incorrect component still occur. There are multiple ways CCV can be implemented and improved by using technology. Researchers have explored digital CCV via barcodes, optical character recognition (OCR), and manual input. When using barcodes or OCR, the system will match the input with a component database. If the correct component is verified, the field worker will be able to continue on with the step. If the correct component is not verified, the field worker should find the correct component before being able to proceed through the procedure. There is always the option to conduct component verification manually, see the right most screenshot in Figure 2.

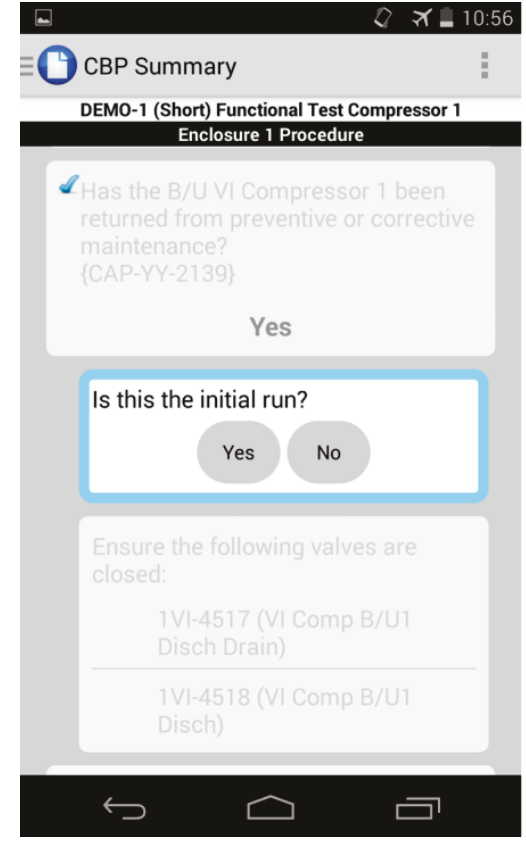

(a) Example of conditional step.

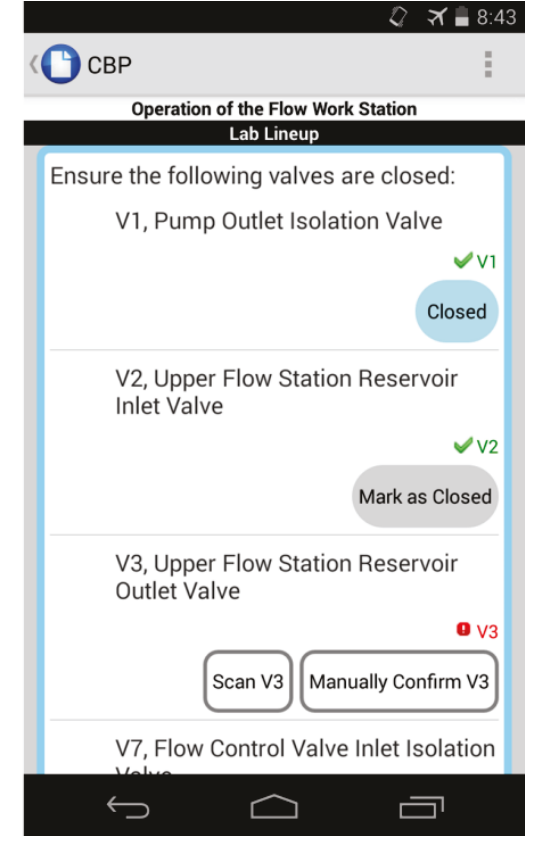

(b) Example of place-keeping and digital $\mathrm{CCV}$.

Figure 2. Screenshots of the basic functionality in the CBP system.

\subsubsection{Procedure Selection and Validation Activities}

In collaboration with PVNGS selected a heating, ventilation, and air conditioning (HVAC) preventative maintenance work order to use for the field evaluation. The work order provides instructions for taking weekly readings from the plant's four HVAC chillers (and related equipment), and for handling out-of-range readings. The original work order instruction contain redundant information, require multiple recordings of values and calculations, and there is no electronic record of the previous recorded values or the conducted procedures. Converting a work order to the AWP system demonstrates the benefits of an electronic work order/procedure system while the technicians are using it in their own plant. The selected work order is conducted weekly for each of the three units at PVNGS.

PVNGS do not have wireless network in the plant. Hence, the researchers had to develop a solution that is independent of wireless communication. In addition, a plant requirement was to keep the devices in airplane mode at all times. The solution was to have all needed information stored locally on the mobile device. 
Multiple talk-throughs with HVAC technicians were conducted to understand the task flow, limitations of the procedure, and how skill of the craft influenced the actual work flow of performing the task. In general, skills of the craft can be thought of as skills that do not require the use of work plan instructions to be performed (EPRI, 2014a). In the context of the AWP research, skill of the craft is defined as a level of skills, knowledge, and abilities required for the individual to perform work safely and effectively based on their qualifications, training, experience, and judgment. Tasks that require a higher reliance on skill of the craft knowledge commonly have less structured (or less formal) instructions than other tasks.

Figure 3 below shows an HVAC technician using the AWP system to conduct the preventive maintenance work order during the field evaluation study.
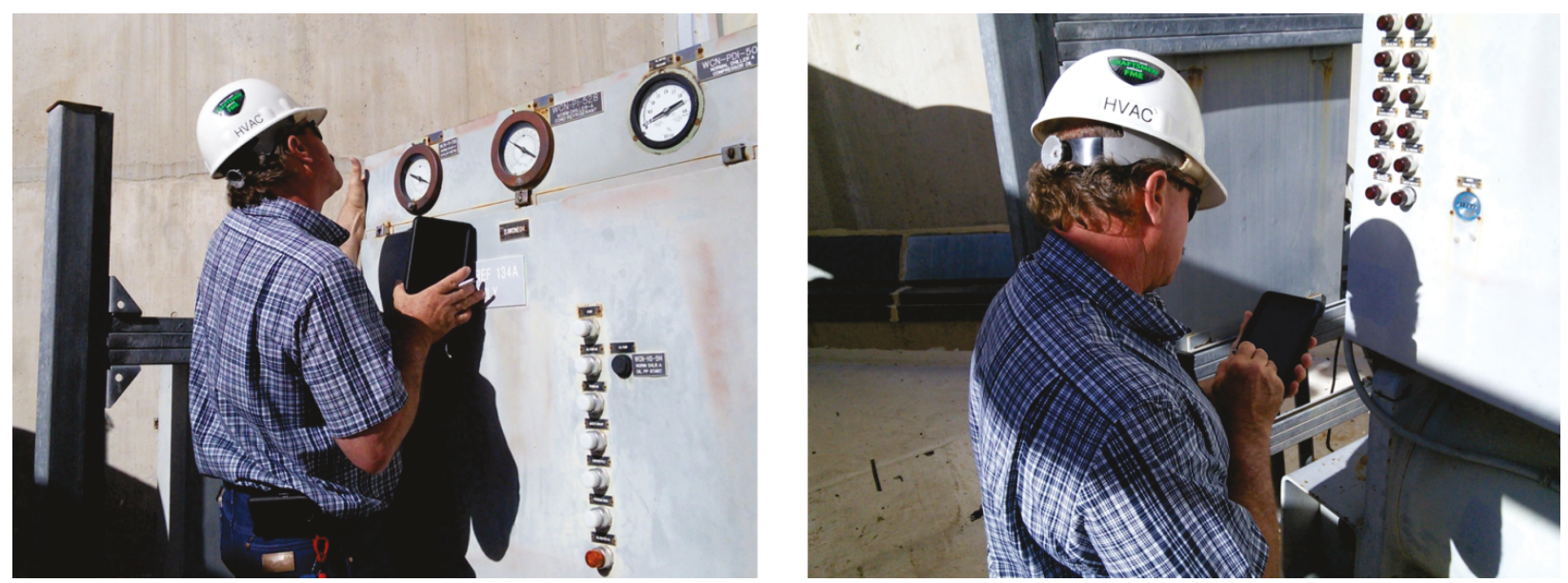

Figure 3. An HVAC technician using the AWP system.

A plant visit was conducted at PVNGS in September, 2014. The goals of the visit were to evaluate the current version of the work order loaded in the AWP system, gather feedback from plant staff, make revisions to the current version if needed, and provide training to the HVAC technicians.

For more detail description of the procedure selection, validation activities, and the overall method used, see Oxstrand and Le Blanc 2015, and Oxstrand et al., 2015.

\subsubsection{Description of the AWP System Used at PVNGS}

As stated earlier, the researchers based the development of the AWP prototype system in the already developed CBP system. The new AWP system encompasses additional functionality to meet the needs of the HVAC technicians. For clarification, even though the figures in this section are screenshots from the AWP system they still have the legacy CBP label in the user interface. As discussed above, the vision for the AWP system is to encompass the whole process related to work packages. CBP is one part of many in this larger AWP system. The researchers decided to build on the existing CBP platform as a starting point in the effort to develop the complete AWP system. For research purposes the researchers decided to keep the CBP label to indicate what part of the overall AWP system is addressed. Below is a description of the functionalities in the prototype system used in the field evaluation at PVNGS.

One of the main new functionalities added to the AWP system was the ability for multiple HVAC technicians to execute a work order across multiple days. To enable this functionality the option to switch users was added to the system as shown in Figure 4 (a). To start a new work order the technician has to create a new instance. This produces the input fields asking for the work order and task numbers, as shown in Figure 4(b). This information is used to create the cover page of the work order (Figure 5). The barcode generated on the cover sheet corresponds to the work order and task numbers, which is the same work process as used for the paper work order except that the barcode is automatically generated in the 
AWP system and the other information on the cover sheet is populated when the technician create the instance.

If the technician is continuing work on an ongoing work order, he/she would first log on to the device. If another technician is already logged in the option to switch logins would be used. The technician would then select the ongoing work order in the menu of instances (i.e., work orders). In the example shown in Figure 4 the only ongoing work order to choose from is "1/O."

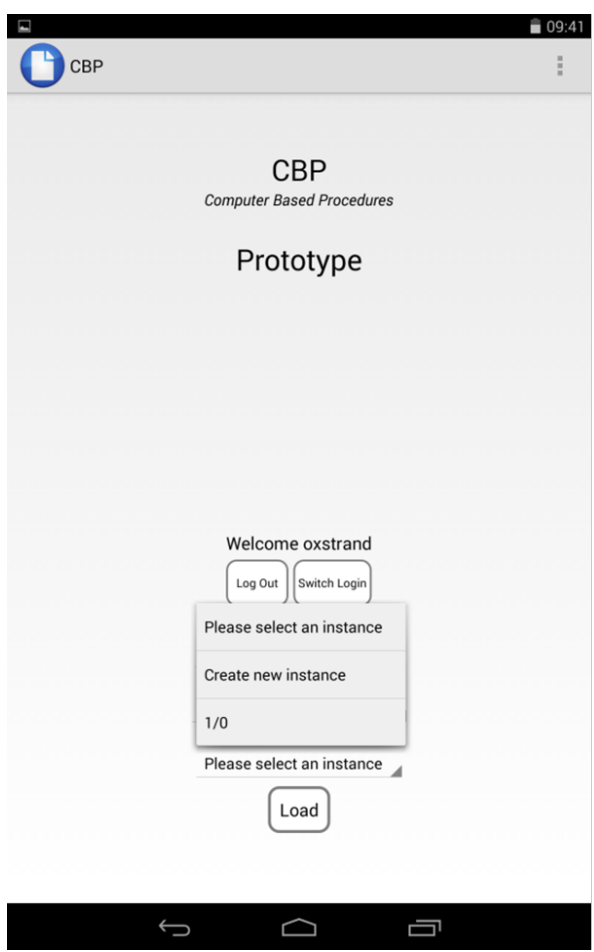

(a) Home screen.

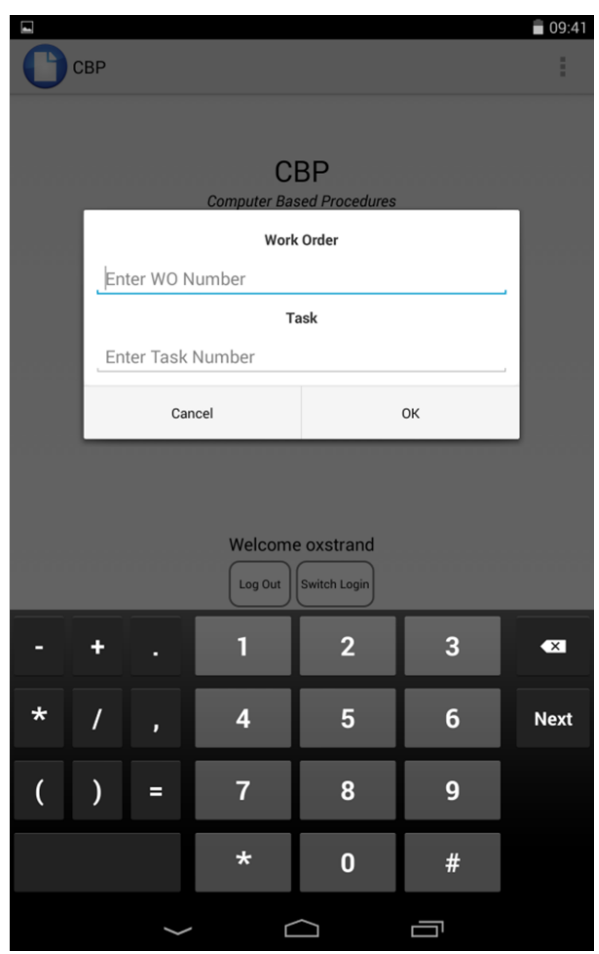

(b) Work order selection.

Figure 4. The home page.

In the background, the system records the identity of the technician that conducted every step of the work order along with the date and time of the taken actions. This information is used for the automatically generated copy of the completed work order. When printed, the automatically generated copy looks very similar to the PVGNS original work order (i.e., it adheres to the plant's formatting template), and it provides the same information as the paper-based work order. For example, the names of the technicians who executed the task, during which time period, which procedure steps were conducted, the outcome of these steps, which steps the technicians marked as not applicable, and all recorded values.

One of the requirements posed by the HVAC preventive maintenance work order was the ability to perform sequence of steps as required by the task. This means that only some parts of the work order could be place-kept in the manner previously designed in the system (i.e., using the blue boarder to indicate active steps, as shown in Figure 6). Figure 7 provides an example of a section, in which steps can be performed in any order. As shown, there is no indication of next applicable step since all the steps qualify for this criterion. Instead, the system displays the action taken, and the technicians cannot close out the work order until all sections are completed. The system indicates to the technician the sections that have not yet been started, the ones that are ongoing, and the ones that are complete. This is illustrated in the rightmost screenshot in Figure 6. 


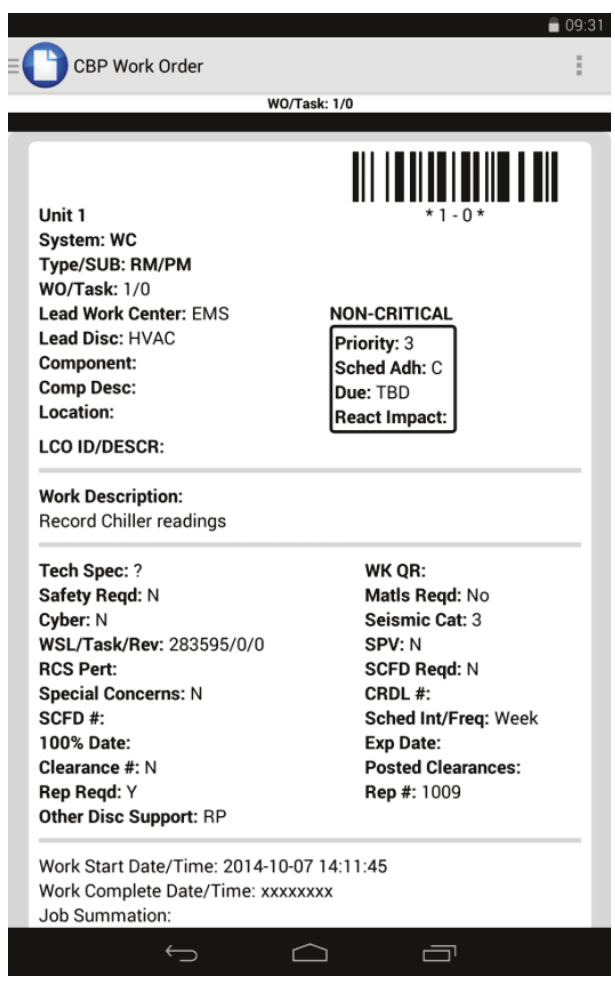

Figure 5. Example of an automatically generated cover page.

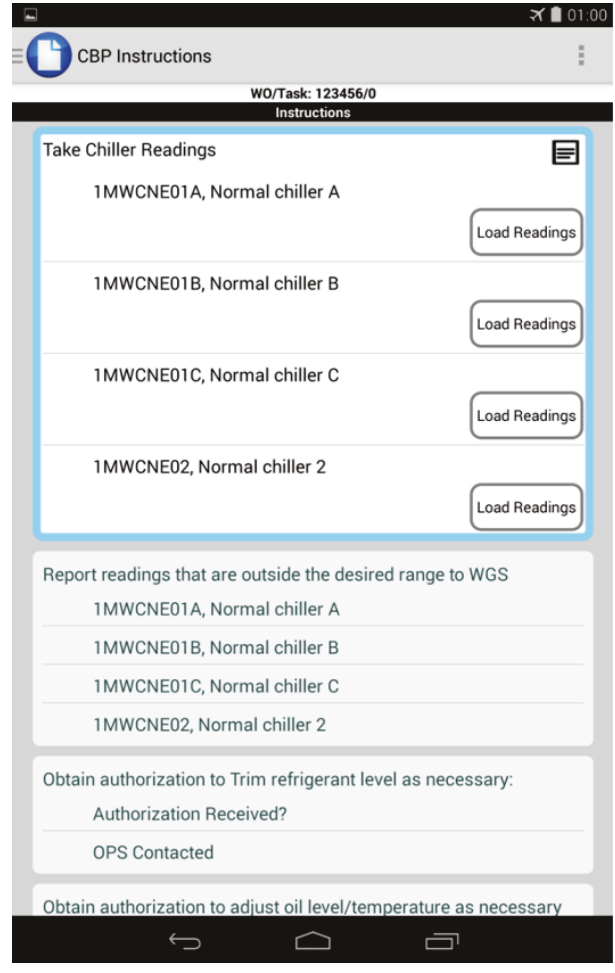

(a) Indication of active step.

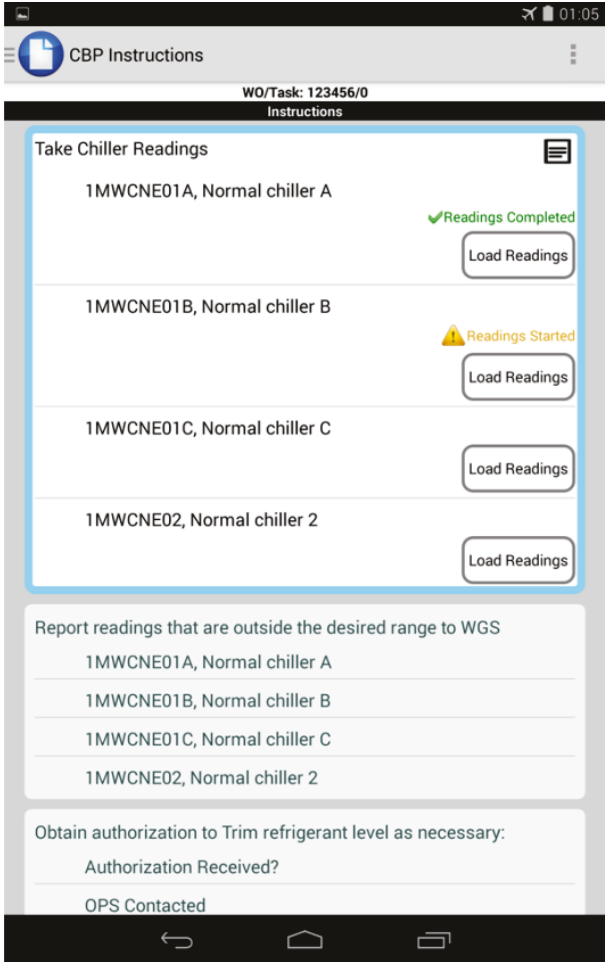

(b) Completed sections and sections in progress.

Figure 6. The place-keeping function. 

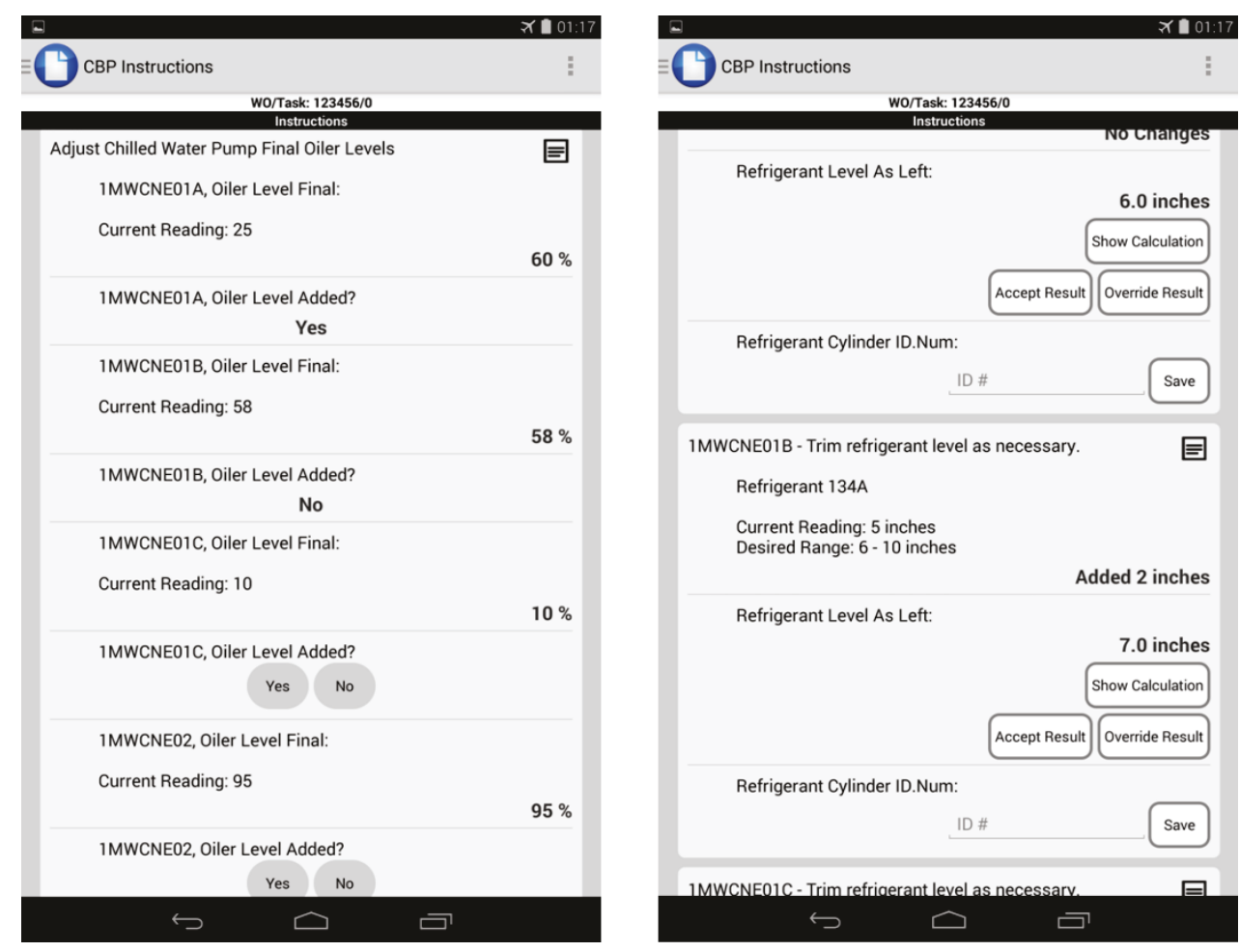

Figure 7. Examples of steps in the HVAC preventive maintenance instruction.

Another function added to the AWP system was the ability to automatically populate the procedure with values from previous logs. Currently, the technicians should go through a binder containing all previous completed work orders to find the last values recorded when the HVAC system was in the same configuration as it is now. By querying an internally located database on the mobile device, the AWP system will find the relevant last logged values and automatically populate them in the relevant steps of the procedure. In the example shown in Figure 8(a) the last recoded value for the 134A refigerant level ( 5 inches) is already inserted in the step. To insert the new reading the technician would click on the text field and the keyboard appears (as shown in Figure 8[b]). To edit previous entered values, the technican would click on the recoded value, which opens up the input field and the keyboard.

The recorded values are currently reported to the engineer on paper. The engineer has to transfer the values to electronic format to log trends. This process includes multiple opportunities for human error and is inefficient and time-consuming. The AWP system exports the recorded values to an electronic format that can easily be shared with the engineer. When the work order is conducted, the technician uses the export function in the system to create a spreadsheet containing all recorded values, which is then emailed to the engineer. 


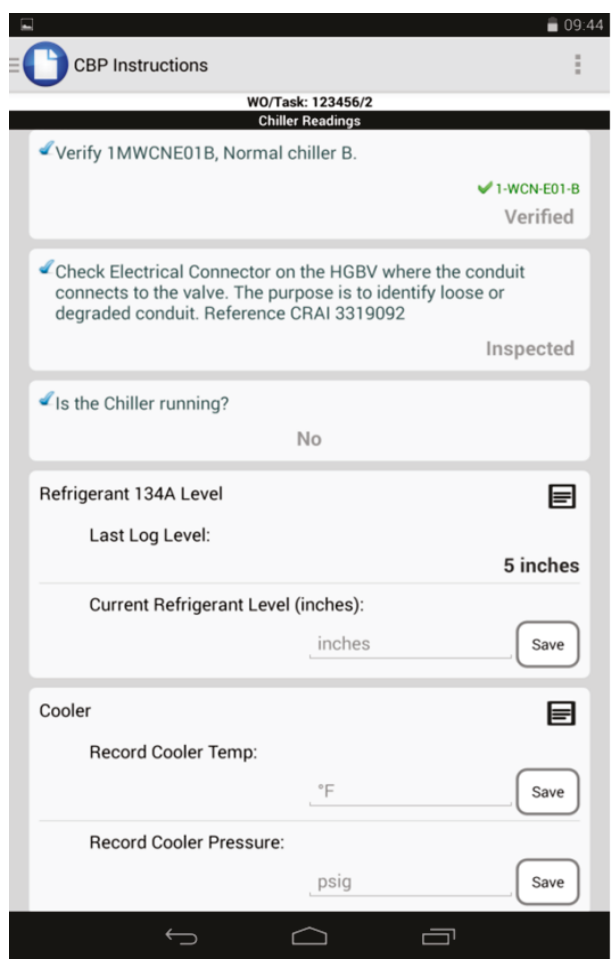

(a) Automated population of last log.

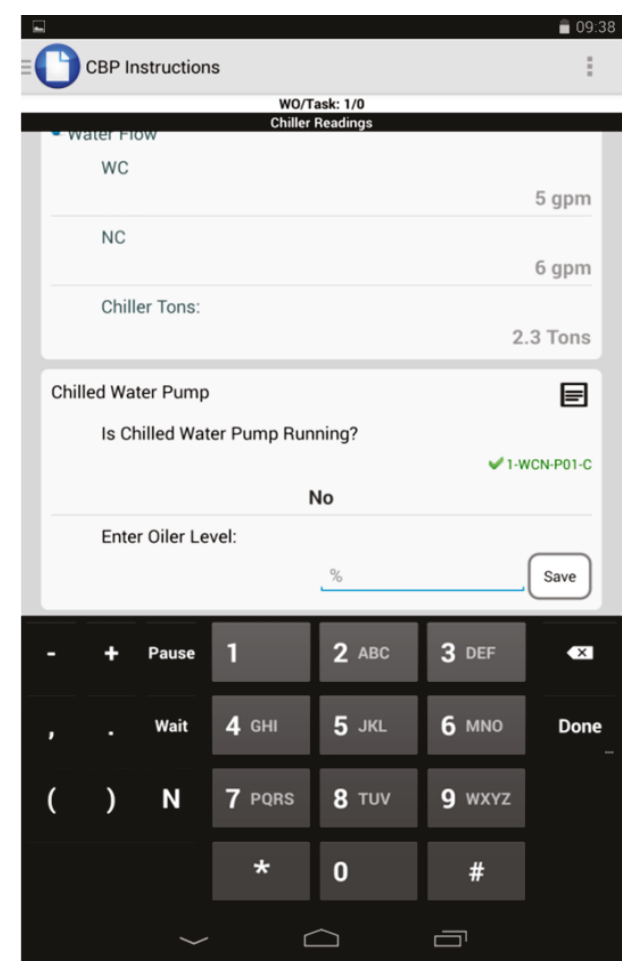

(b) Recording of value.

Figure 8. Example of how to record readings.

Findings of the field studies conducted in the LWRS CBP project shows that calculation errors or mistakes are quite common when a paper procedure is used to perform a task. For example, users might use the wrong formula, make an error in the calculation, or make rounding errors. When users conducted the same task using a computerized tool (in this case a CBP system, the system automatically calculated the value based on a single input, completely preventing this type of error (Le Blanc et al., 2015, and Oxstrand et al., 2014). The researchers used the findings in the previous research and designed the AWP system to automatically conduct calculations based on the readings and other relevant user input, which prevents any human calculation errors. The technician has the option to review the calculation that lead to the calculated value. To do so, the technician would use the "Show Calculation" option shown in Figure 9(b). Before moving on to the next step the technician has to either accept the calculated value or to override the calculated value. To override the technician is required to both provide the new value and to provide a reason for the overriding action (see Figure 9(a). This information is stored and presented in the final copy of the completed work order.

To provide good visual representation of the overall performance of the plant system the option to generate trend graphs based on the recorded values was added to the AWP system. Figure 10 provides an example of such graph. The specific graph in the example shows the trend for the cooler water inlet temperature under the condition that the chiller is running.

The navigation within the work order was made easy by the new slide-out menu. The menu is accessed either by sliding your finger from the left side of the screen towards the middle (see red arrow in Figure 11) or by clicking on the icon in the upper-left corner as indicated by the red circle. The slide-out menu provides options to navigate to the home screen, the work order summary, and to the procedure steps (i.e., the instruction part of the work order). 


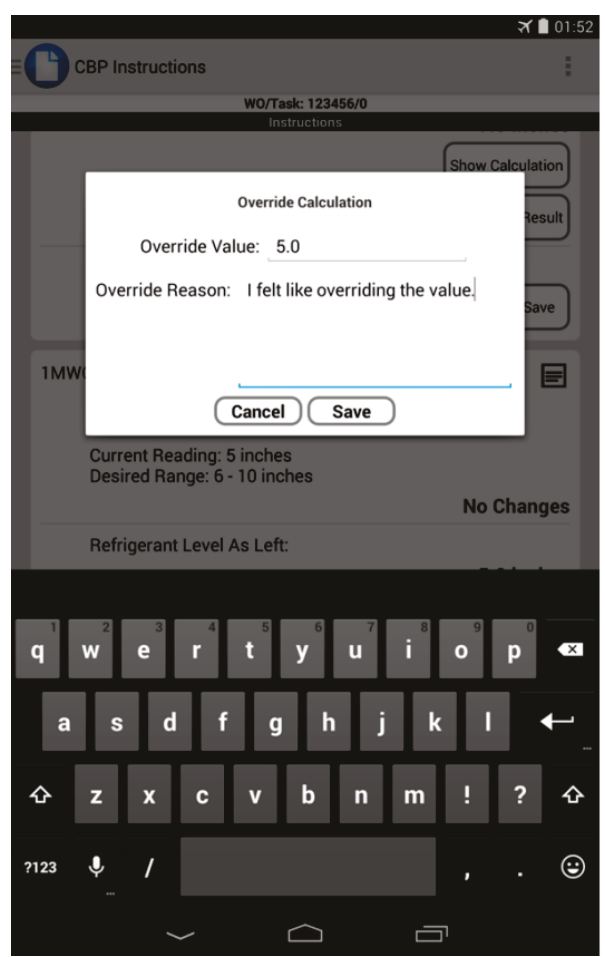

(a) Pop-up for overriding calculation.

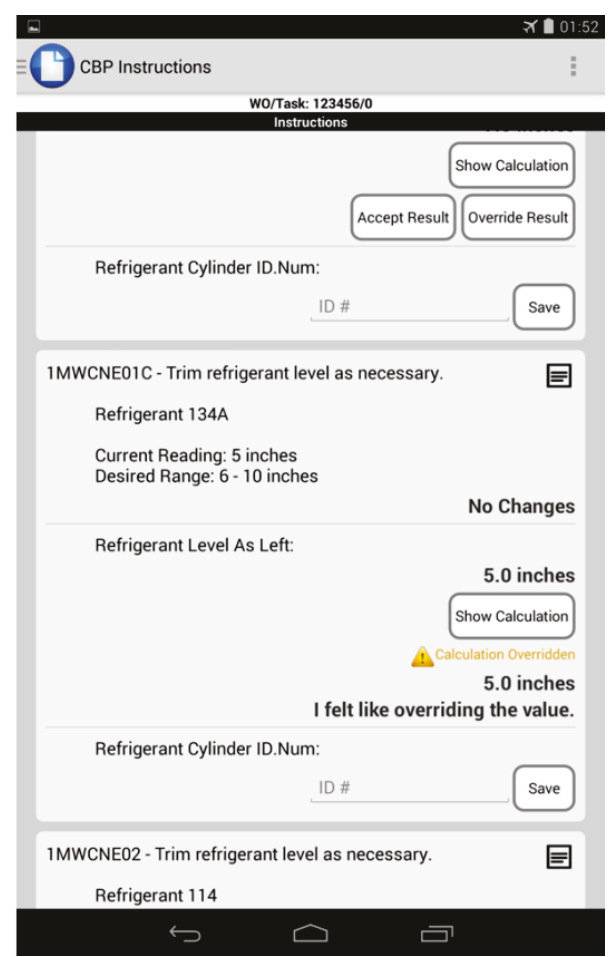

(b) Visual representation of overridden calculation.

Figure 9. Overriding a calculation.

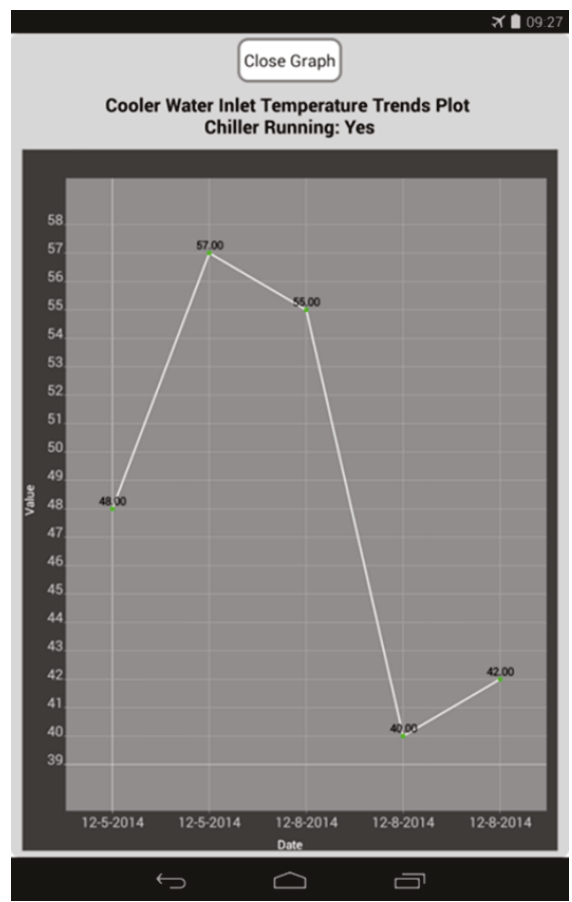

Figure 10. Example of trending graph. 


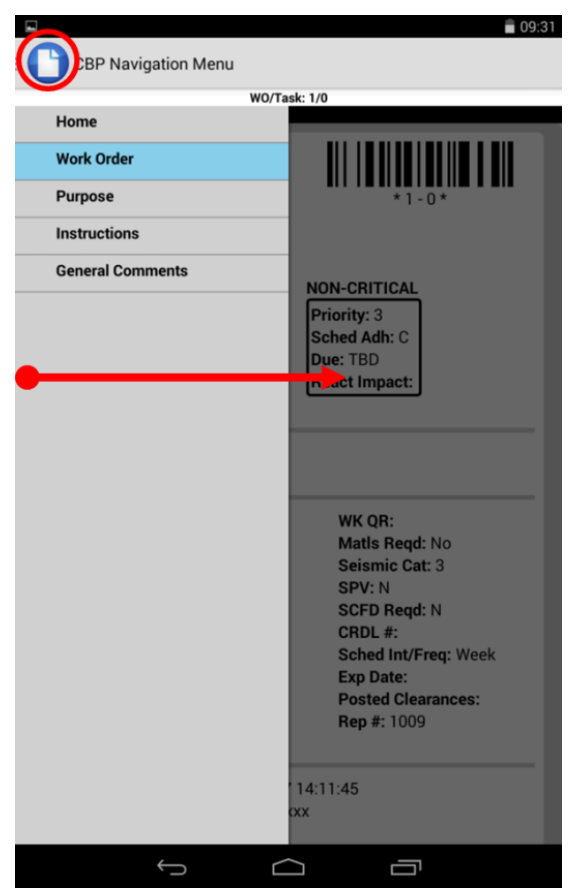

Figure 11. Navigation by slide-out menu.

The technicians sometimes need to take notes related to a specific step in the procedure. This is commonly done by writing the note in the margin of the paper procedure. The researchers added the note functionality to the AWP system that can be accessed in two ways. The technician has the option to take a note directly related to a step in the procedure as well as the option to write notes that are generic to the whole work order. Both options are presented in Figure 12 where the screenshot (Figure 12[a]) shows the icon for taking notes associated with a specific step. The screenshot (Figure 12[b]) shows the slide-out menu option for making general notes or comments.

\subsubsection{PVNGS Field Evaluation Method}

The participants in the PVNGS field evaluation study include the HVAC technicians tasked to conduct the HVAC preventive maintenance work order each week throughout the duration of the study. During the validation activity, the research team provided training on the AWP system to the HVAC involved technicians. These technicians were then instructed to train their peers on the AWP system. The duration of the study was initially planned to be 26 weeks long, but in the end it lasted 23 weeks between September 2014 and February 2015.

A web-based survey was developed and the participants were asked to fill it out after completing the preventive maintenance work order with the AWP system. The goal of the survey was to assess the usability of the AWP system and device. The survey was also developed to gain more detailed feedback on the design of the user interface and the overall experience using the computer-based work order. Three 7-inch Nexus 7 tablets were used in the study (one per unit at the plant) with the HVAC preventative maintenance work order loaded on each tablet. 


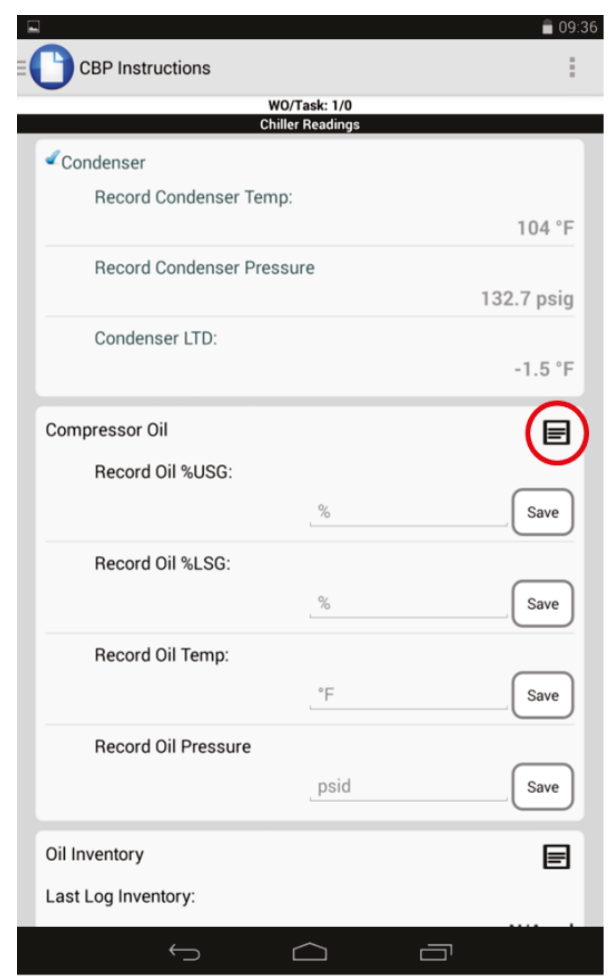

(a) Note associate with step.

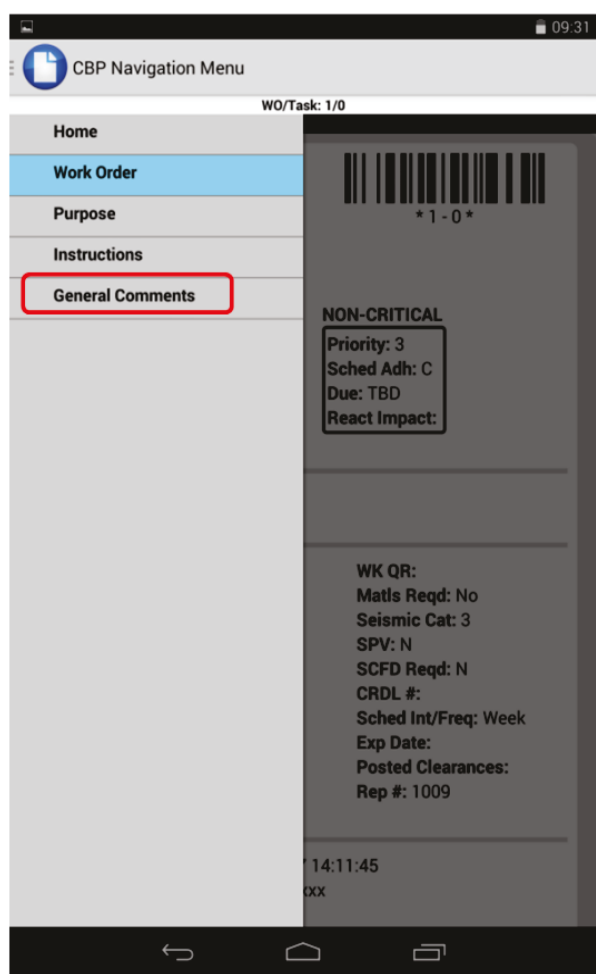

(b) General comments.

Figure 12. Note taking functions.

\subsubsection{Discussion of Results}

The feedback provided by the HVAC technicians was compiled and analyzed by the research team after the study officially ended and the devices were returned to INL. The results can be summarized in two major points;

- Skill of the craft knowledge does not translate well into an automated CBP system

- Load and response times impact the usability of the system

The specific work order selected for the PVNGS field evaluation study requires a large amount of skill of the craft knowledge. For example, the procedure did not fully specify which steps or conditions required actions to be taken or what these actions would be. The amount of reliance on skill of the craft knowledge varies between work orders, organizations within the plant, and between utilities. It will be up to the utility to decide (1) the accepted level of reliance on skill of the craft knowledge, and (2) how much of this type of knowledge should be proceduralized. The result of the field evaluation study shows that a high reliance on skill of the craft knowledge makes it difficult to adequately design context sensitive instructions. In comparison, converting a more structured procedure and work flow into the format used by the AWP system results in a more straightforward activity. The challenge with work orders such as the HVAC preventive maintenance work order is to predict all possible ways a technician could potentially conduct the task.

To adequately handle the level of task flow autonomy requested by the technicians the researchers had to revise the AWP system to provide the ability to perform allowed sections and steps in any order. However, this added a new level of complexity to the system, in terms of the amount of cross-checks and decisions updates made by the system, which in turn made the AWP system run slower than previous iterations of CBP and AWP systems. The load and response times became seconds long compared to less than a second. Even though the HVAC technicians expressed how much they liked the ability to use the 
AWP system to perform the task in the order they preferred, the longer load and response times did have a negative impact on the overall user experience. One important lesson learned is the importance of load and response times in terms of user acceptance of a system.

\subsection{Field Evaluation at Southern Nuclear Company Plant Vogtle}

In the early summer of 2015 a field evaluation study was launched at Southern Nuclear Company's plant Vogtle, Units 1 and 2. The field evaluation study builds on the lessons learned from the previous AWP field evaluation study at PVNGS in 2014/2015.

\subsubsection{Procedure Selection and Validation Activities}

A plant visit was conducted in March 2015. During the visit the research team and plant personnel decided to use the "battery and charger test and inspection maintenance" work order for the study. After the plant visit, the battery and charger test and inspection maintenance work order was revised and split into nine separate work orders: one per A to D train for both units and one for inspection of non-safety related batteries and chargers. The researchers decided to include the eight instructions for safety related inspections in the study. The maintenance organization at Vogtle conducts one of the nine work orders every week on a rotating schedule.

As a part of the plant visit, the researchers observed the battery and charger test and inspection task being conducted. This provided insights on who is involved in the task, required actions, areas in the task flow that works well, and areas in the task flow that could potentially be improved by the AWP system. The task is typically performed by two maintenance technicians: one is taking the readings and one is completing the data sheet and place-keeping the procedure. Coordination was also necessary because some steps are required to be carried out by an operator rather than maintenance technicians. The operators are the only group allowed to take action on the plant, hence steps to turn on/off equipment has to be conducted by operations. Examples of items to be improved by the AWP system are the frequent flipping between the instruction set and the associated data sheets as well as ways to ensure the work order is conducted on the correct train in the correct unit. Figure 13 shows a maintenance technician performing the battery and charger test and inspection work order.

During the plant visit, the researchers and plant personnel focused on filing the completed work order. In other words, the focus was on means to streamline and improve the process of filing and archiving the completed task. The current process consists of ensuring all signatures and inserted data points are recorded on all pages where they are required, creating an electronic copy of the work order by scanning all the pages of the procedure, attaching a cover sheet, and finally submitting the package to the internal archiving system. The majority of these tasks can be verified and carried out automatically by the AWP system.

A second plant visit was conducted in June, 2015. The researchers met with the procedure writer responsible for the battery and charger test and inspection, a representative of the Information Technology (IT) group, a maintenance supervisor, and two maintenance technicians. The maintenance supervisor and technician reviewed the computerized version of the work order and compared it to the original paper version. The goals of the review were to ensure the version in INL's AWP system met the intent of the original version, and to identify any editorial mistakes and task flow inefficiencies. Editorial and task flow revisions were addressed during the plant visit and the revisions were validated by the technicians and the procedure writer. The IT representative played an essential role in getting the mobile devices approved to be used in the plant, and to get the AWP system application and the post-task survey loaded on a work station in the maintenance office. 

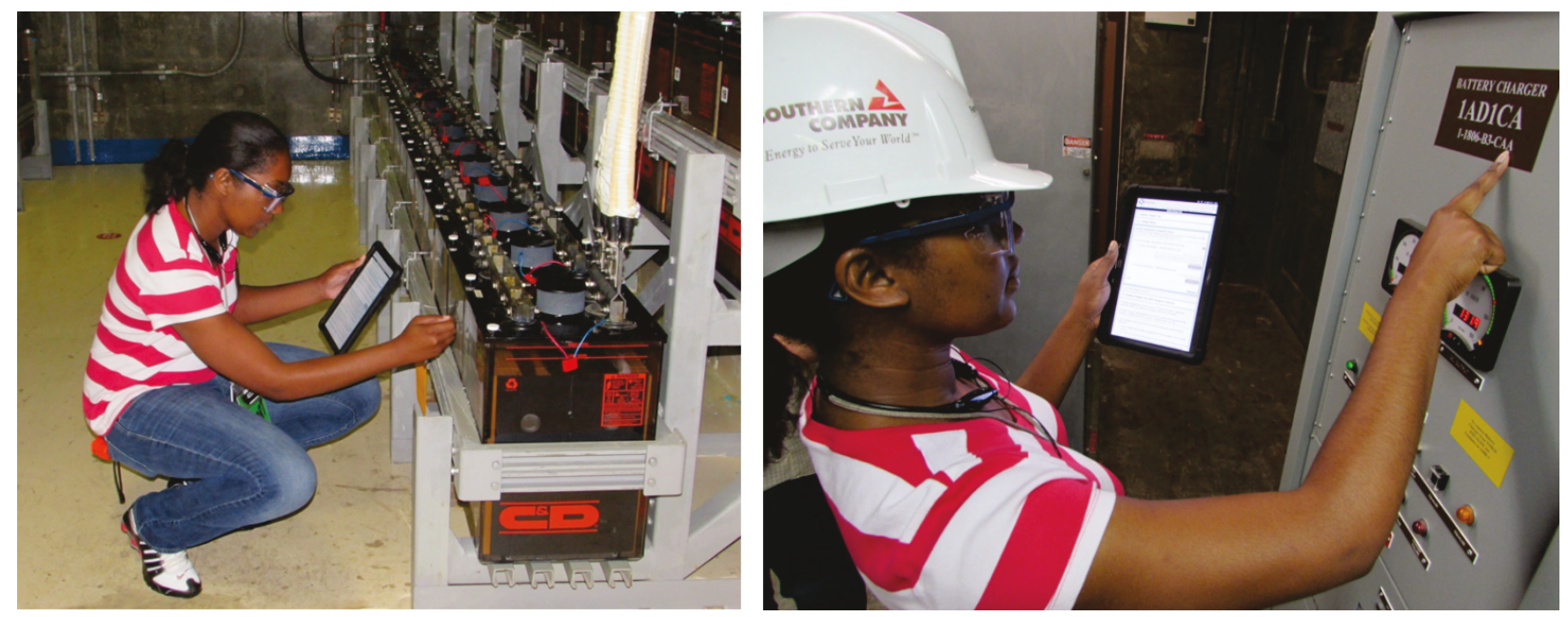

Figure 13. A maintenance technician performing the battery and charger test and inspection work order.

Two different mobile devices were used, one Samsung 10-in. tablet and one Nexus 7-in. tablet. The maintenance technicians used both devices to conduct walkdowns of the task in the plant. The walkdowns were designed to provide training of the system to the technicians and to verify that the computer-based version of the work order met the actual work flow of the task. In other words, the researchers aimed to make sure the work flow prescribed by the AWP system met the practical aspect of task execution, and that it did not introduce any process inefficiencies.

\subsubsection{Description of the AWP System Used at Vogtle}

A couple of new functionalities were added to the AWP system, which are described below. In addition, the system as a whole went though many minor improvements to the user interface. This section will provide an overview of the most current design concepts of the AWP system used for the field evaluation study at Vogtle 1 and 2.

The navigation within the work order is very similar to the version used for the PVNGS study (i.e., by using the slide-out menu). The main change to the menu is the option to navigate straight to a specific section of the work order, as shown in Figure 14. The section in which the current relevant step is located is highlighted in blue. The home screen is the very first screen the technician sees when starting the AWP system. This is where the technician logs into the AWP system and selects the work order. This is also the screen used to navigate between assigned work orders and/or when there is a need to switch users (e.g., at a shift turn-over).

When the technician is logged on to the system and has selected a work order to perform, the technician is taken to the summary page. This is where all sections of the work order shows up until the point of the actual instructions are presented to the technician. The sections presented on the summary page are performed before the technicians walk to the work location in the plant. As shown in Figure 15(a), an additional step was added to the summary reminding the user to ensure the device is in airplane mode. The step was added upon request to mitigate the risk as the device unintentionally was left in wireless mode as the technicians entered the work location. 


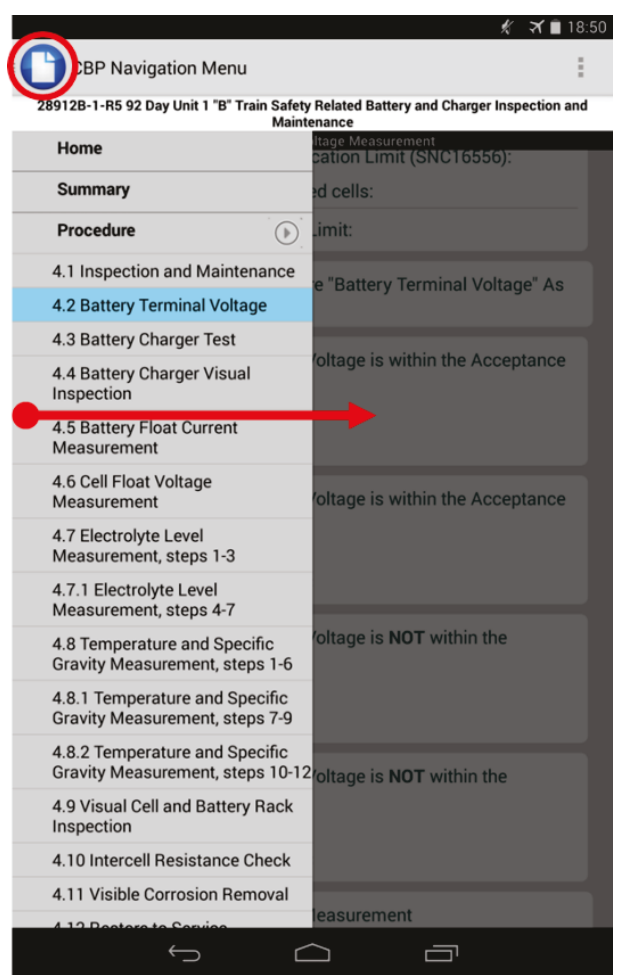

Figure 14. Example of the revised slide-out menu.

Most sections on the summary page require place-keeping (i.e., steps should be acknowledged, ensured, or signed). Visual cues are presented to the user as a reminder of the required action, see Figure 15(b). The action verbiage changes tense upon clicks of the button. For example, "Acknowledge" changes to "Acknowledged" and "Ensure" changes to "Ensured." In addition, the color of the place-keeping function changes from gray to blue. These design concepts provide visual cues to the technician about actions to take and actions taken, and hence provides an overview of the technician's progression though the summary page.

The technician can review the procedure while still working on the summary sections. However, no actions can be taken on the procedure until all the sections on the summary page are completed (i.e., adequately place-kept). This is indicated in Figure 16(a) the screenshot requires the technician to obtain the shift supervisor's authorization prior to performing the work. As soon as the authorization is obtained and the step is signed off, the option to start the procedure becomes visible (see Figure 16[b]). 


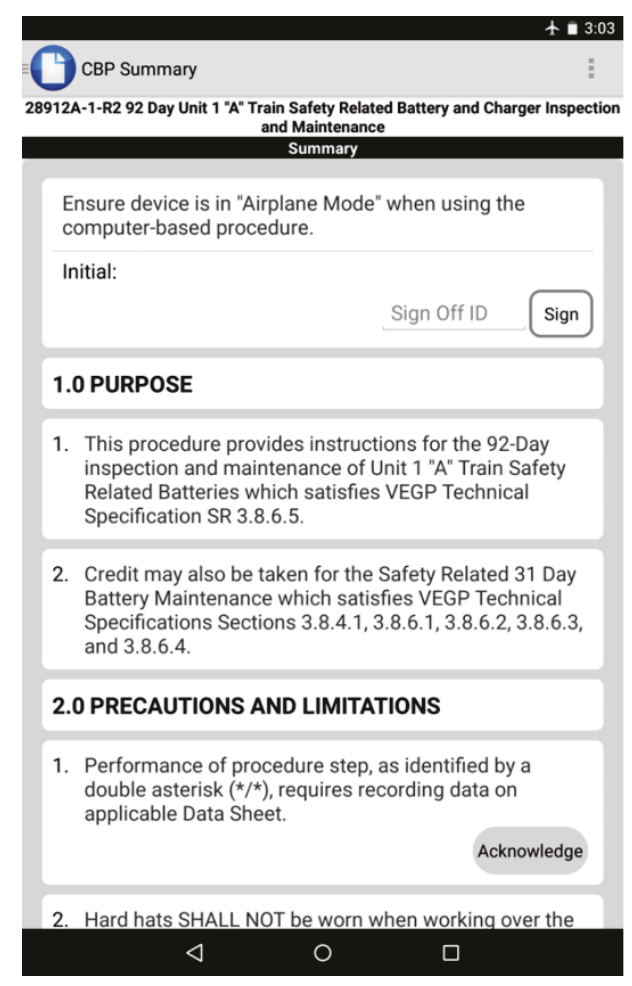

(a) Airplane mode step.

Figure 15. Example of the summary page.

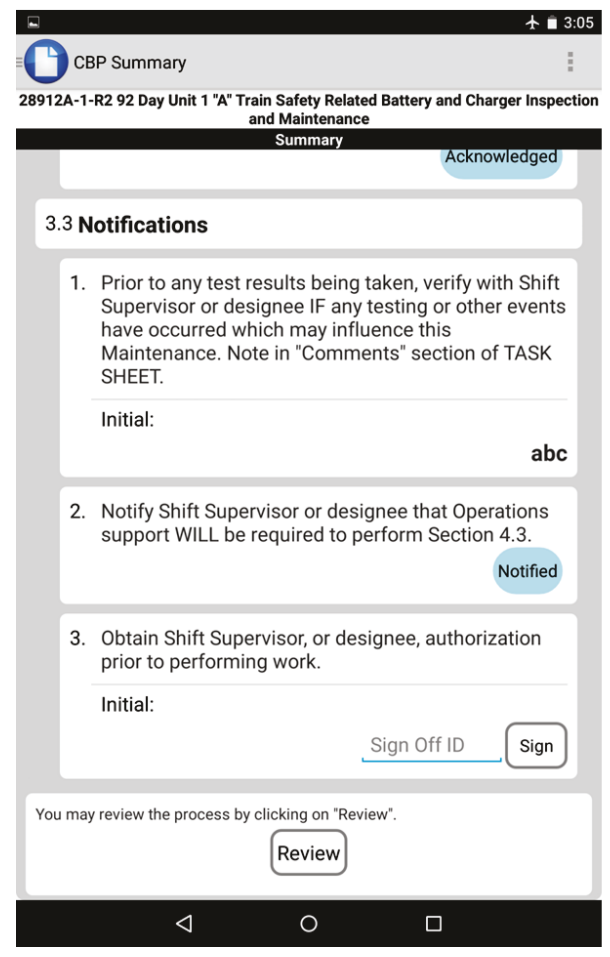

(a) Review option while front matter is in-progress.

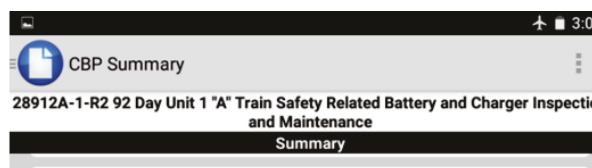

2. Hard hats SHALL NOT be worn when working over the batteries.

Acknowledged

3. Do NOT place any material or equipment on top of battery cells.

Acknowledge

4. IF any discrepancies are encountered that CANNOT be corrected within the scope of this procedure, submit a Condition Report to initiate further corrective action. The CR number will be recorded in the "Comments" section of the Attachment 2, TASK SHEET.

Acknowledged

5. Ensure no work activity to preclude circuit interruption or arcing in the vicinity of the battery cells. (SNC11225)

Ensured

6. Ensure a neutralizing agent for acid spills is available.

Ensure

7. Before touching the battery, discharge "body static" electricity by touching a grounded surface

$$
\triangleleft \quad \bigcirc \quad \square
$$

(b) Place-keeping of procedure front matter.

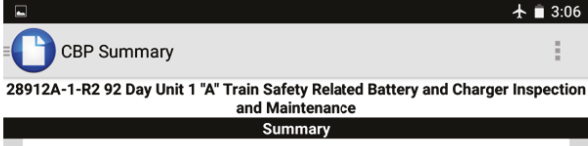

3.3 Notifications

1. Prior to any test results being taken, verify with Shift Supervisor or designee IF any testing or other events have occurred which may influence this Maintenance. Note in "Comments" section of TASK SHEET.

Initial:

2. Notify Shift Supervisor or designee that Operations support WILL be required to perform Section 4.3 .

Notified

3. Obtain Shift Supervisor, or designee, authorization prior to performing work.

Initial:

You may review the process by clicking on "Review" or you may start it by clicking on "Start".

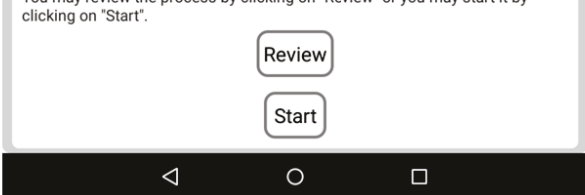

(b) Start option.

Figure 16. Example of options to preview and start procedure. 
The specific work order used for the field evaluation study at Vogtle 1 and 2 required that four of the 59 battery cells were thoroughly inspected. These pilot cells are determined before the technicians are assigned the work order. The pilot cell information is currently communicated in the task preview and pre-job brief. As a reminder, the technician marks the data sheets of the paper copy of the work order accordingly, as indicated by the asterisks in Figure 17. For each step in the procedure where actions need to be taken on the pilot cells, the technicians should make sure to use the right pilot cell numbers and iterate the specific steps four times (ones per pilot cell).

\begin{tabular}{|c|c|c|c|c|c|c|}
\hline $\begin{array}{l}\text { Cell No. } \\
\text { Asterisk the } \\
\text { pilot cells }\end{array}$ & $\begin{array}{l}\text { Measured } \\
\text { Cell Float } \\
\text { Voltage } \\
\end{array}$ & $\begin{array}{c}\text { As Found } \\
\text { Electrolyte } \\
\text { Level }\end{array}$ & $\begin{array}{c}\text { As Left } \\
\text { Electrolyte } \\
\text { Level }\end{array}$ & $\begin{array}{l}\text { Measured } \\
\text { Cell Temp }\end{array}$ & $\begin{array}{c}\text { Measured } \\
\text { Specific } \\
\text { Gravity } \\
\end{array}$ & $\begin{array}{c}\text { Corrected } \\
\text { Specific } \\
\text { Gravily } \\
\end{array}$ \\
\hline 40 & 2. 23 & $-1 / 8$ & $\mathrm{NA}$ & NR & 1. $\mathrm{dA}$ & 1. NA \\
\hline 41 & 2. 24 & - & & $\downarrow$ & 1. & 1. \\
\hline $42 \quad *$ & 2. 24 & $-\sqrt{1}$ & & $78^{\circ}$ & 1. & 1. \\
\hline 43 & 2. 24 & $-1 / 4$ & & NR & 1. & 1. \\
\hline 44 & 2. 24 & -1 & & & 1. & 1. \\
\hline 45 & 2. 24 & $-\quad 1 / 4$ & & & 1. & 1. \\
\hline 46 & 2. 25 & $-1 / 8$ & & & 1. & 1. \\
\hline 47 & 2. 25 & - & & & 1. & 1. \\
\hline 48 & 2. 24 & -5 & & & 1. & 1. \\
\hline 49 & 2. 24 & $-\quad 1 / 4$ & & & 1. & 1. \\
\hline 50 & 2. 24 & -1 & & & 1. & 1. \\
\hline 51 & 2. 24 & 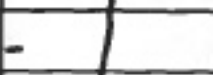 & & & 1. & 1. \\
\hline 52 & 2. 23 & - & & & 1. & 1. \\
\hline 53 & 2. 24 & $-\quad t$ & & & 1. & 1. \\
\hline 54 & 2. 24 & $-1 / 8$ & & & 1. & 1. \\
\hline 55 & 2. $y$ & -1 & & & 1. & 1. \\
\hline 56 & 2. 24 & $-\quad 1 / 4$ & & & 1. & 1. \\
\hline 57 & 2. 24 & -1 & & 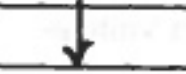 & 1. & 1. \\
\hline 米 & 2. 24 & $-1 / 8$ & & $78^{\circ}$ & 1. & 1. \\
\hline 59 & 2. 24 & $-1 / 4$ & 监 & NA & $1 . \downarrow$ & $1 . \downarrow$ \\
\hline
\end{tabular}

Figure 17. Example of mark-up of pilot cells in the data sheets (cell 42 and 58 are pilot cells here).

To improve the process of the pilot cells in the AWP system, the researchers added an initial step in the procedure asking the technician to record the four pilot cell numbers, as shown in Figure 18. This information is stored in the system and used throughout the execution of the battery test and inspection. The AWP system uses the recorded pilot cell numbers to direct the technician to take specific actions on these cells rather than present a generic step for all four (unnumbered) pilot cells. 


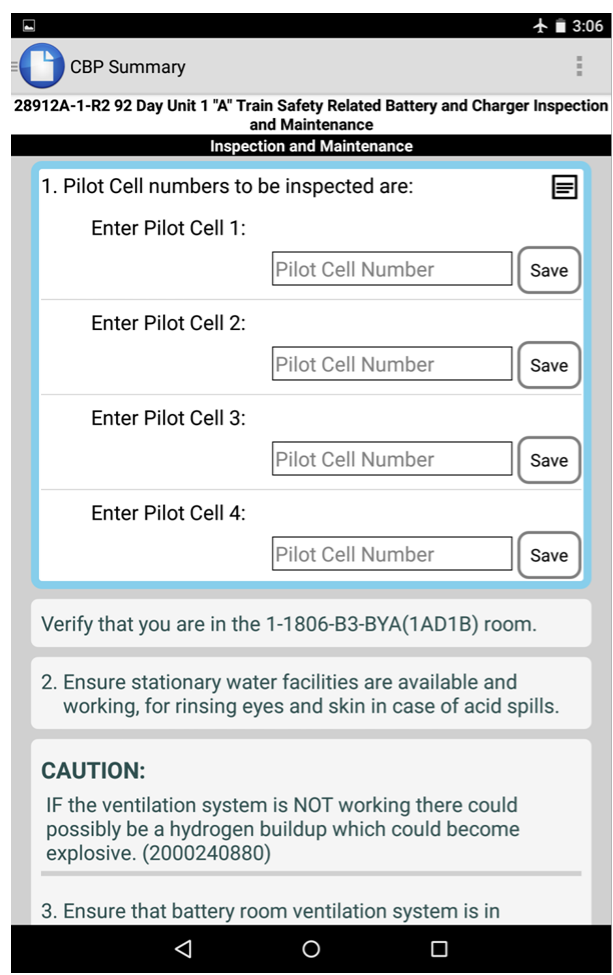

(a) Input fields for pilot cells

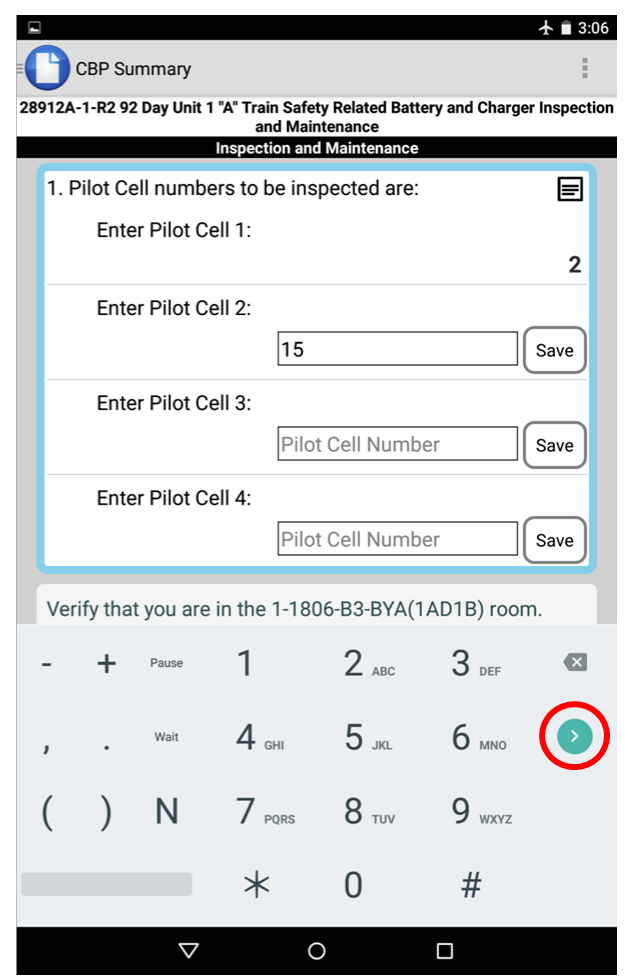

(b) Improved user experience of recording multiple values

Figure 18. Determining pilot cells.

The dynamic use of recorded data to direct the technicians through the execution of the task is one of the main strengths of the AWP system compared to the paper version of work orders and procedures. During the plant visits, the researchers observed a large amount of time spent going between the procedure and the attached data sheets. These sheets are used to combine multiple readings from each of the 59 batteries, such as voltage readings. Using the AWP system all the readings still need to be recorded; however, the data sheets are automatically populated and the recorded data points are used to automatically verify that values are within range and/or meet technical specification requirements.

One additional improvement made to the AWP system before launching the Vogtle field evaluation study was to improve the user experience of recording values. Two features made a noticeable difference on the user experience and efficiency. These are automatically moving to the next text field and reduced amount of text to record. In earlier versions of the system the technician had to type in the value, click save, click on the next input field, and type in the next value. Now, the technician types the value and clicks "next" (arrow indicated by the red circle in Figure 18[b]). The cursor is automatically moved to the next text field. If there are no more fields, the step is marked as completed. As shown in Figure 19(b), many of the values recorded are very similar. In the example in Figure 19, all values are in the format of 2.XX, hence the integer part of the value is always 2 in normal conditions. Knowing this information, there is no need to ask the technicians to type the integer and decimal point for each of the 59 batteries. In the example of Figure 19, the technician only recorded the fraction valves of 22 or 23 for the different cell voltages instead of typing 2.22 and 2.23. In other words, it is 118 characters the technician does not have to type on the keyboard in this one procedure step. This will become quite time saving over the course of a task execution that requires multiple readings for a large amount of components, such as the Vogtle work order. 

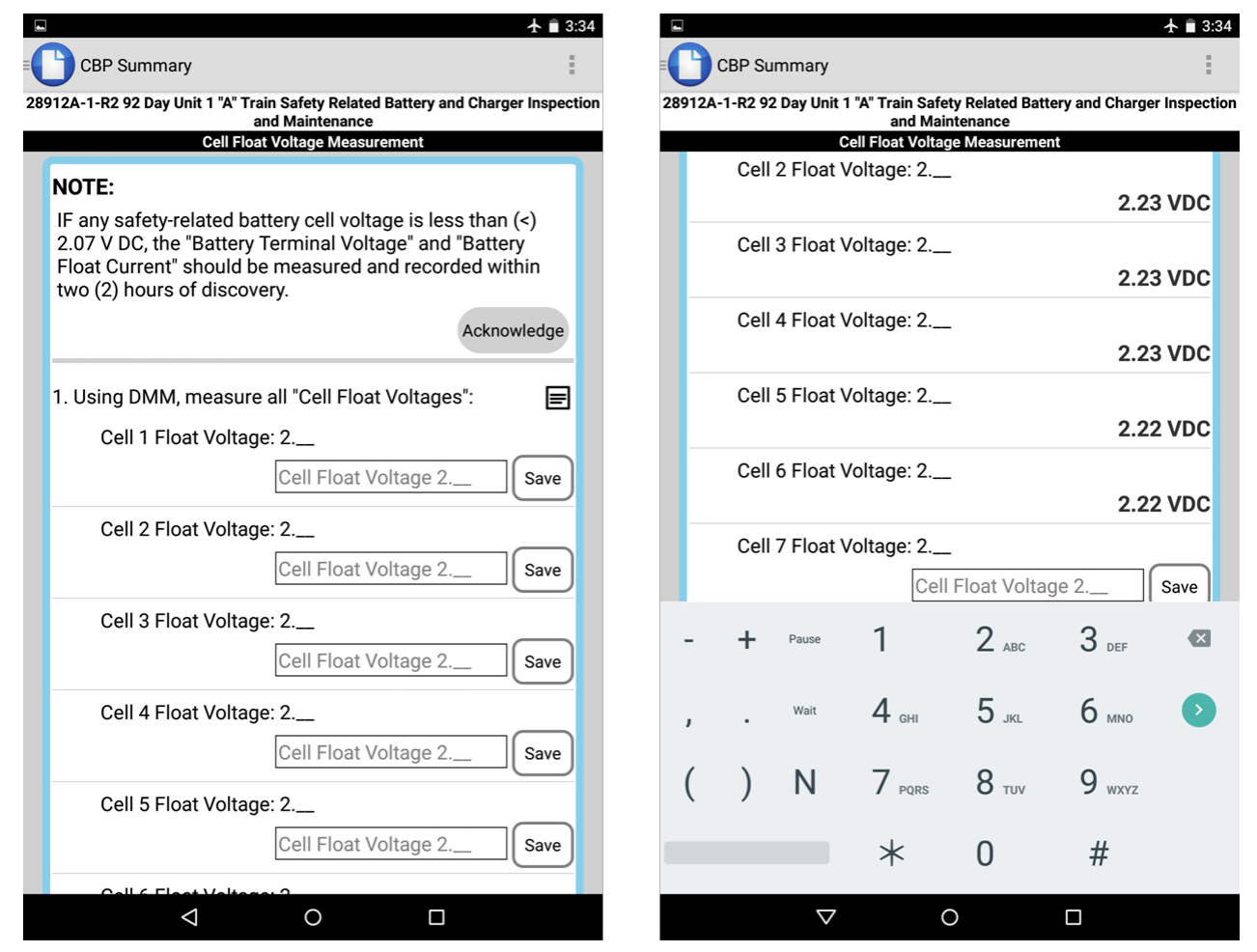

Figure 19. Example of improved recording of readings.

If needed, the technician can revise already-recorded values by clicking on the value. This will open the text field and bring up the keyboard. If an already-completed step needs to be revised, the technician uses the checkmark in the upper-left corner of the step to be revised (see Figure 20). This produces a prompt asking if the step should be revised. If yes, the step will be opened up in editing mode. Depending on the step, the technician can change recorded values and/or decisions points. When the step is edited and saved, the procedure flow will be updated to reflect the changes.

The screenshot in Figure 20(a) shows how the AWP system simplifies the presentation of step logic to the technician. Conditional statements are rephrased as binary questions, for example the statement "IF any cells are jumpered, THEN perform..." is presented as the question "Are any cells jumpered?" The simplified step logic streamlines the information and reduces the technician's cognitive workload. The presentation of a conditional step is presented in the screenshot shown in Figure 20(b) (Step 2). Observe in Figure 20(b), there are no jumpered cells and the recorded value is out of the accepted range. The battery terminal voltage recorded in Step 3 did not meet the acceptance criteria in the technical specification. The AWP system indicates this to the technician by presenting the technical specification limit along with the recorded value.

The collaboration partners at Vogtle mentioned to the research team that, just like the industry as a whole, Vogtle has been struggling with incidents of actions being conducted on incorrect component and in some cases even in the wrong unit. The AWP system incorporates a tool to prevent these incidents (i.e., the digital CCV). There are two different options to conduct digital CCV in the AWP system; barcodes and OCR. Both methods were tested and prepared to be used during the Vogtle study. However, during the walkdowns it was concluded that there are some discrepancies between labels used in different trains and in the different units. These discrepancies could potentially render the OCR option unreliable in the sense that it potentially would misinterpret the labels and therefore not approve the component verification. For the technicians to want to use the AWP system it is important that the amount of unreliable technology is kept at a minimum. Generally, users prefer systems that behave in a predictive manner. Therefore, it was decided to disable the OCR option. 


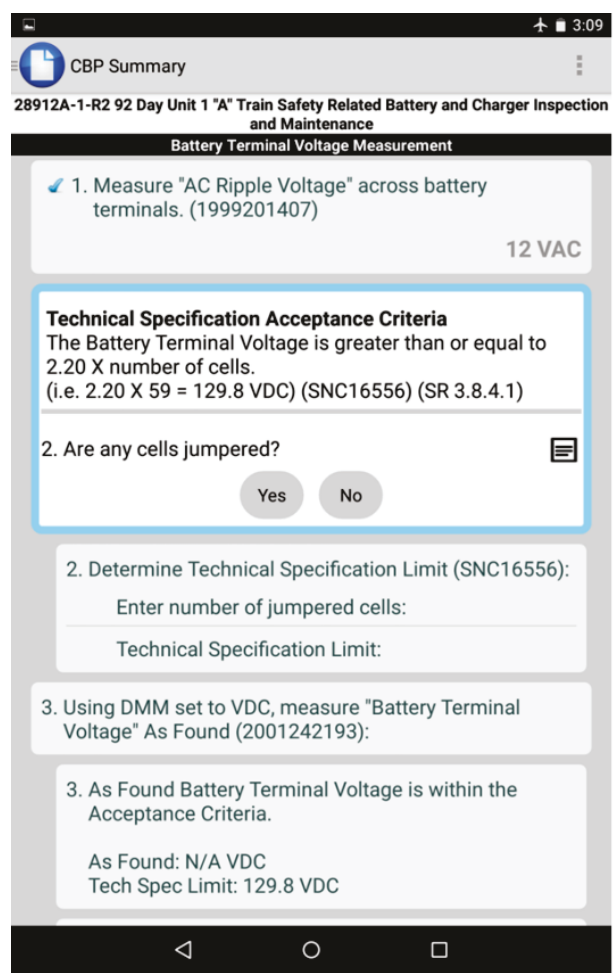

(a) Tech spec acceptance criteria example linked to step and simplified step logic.

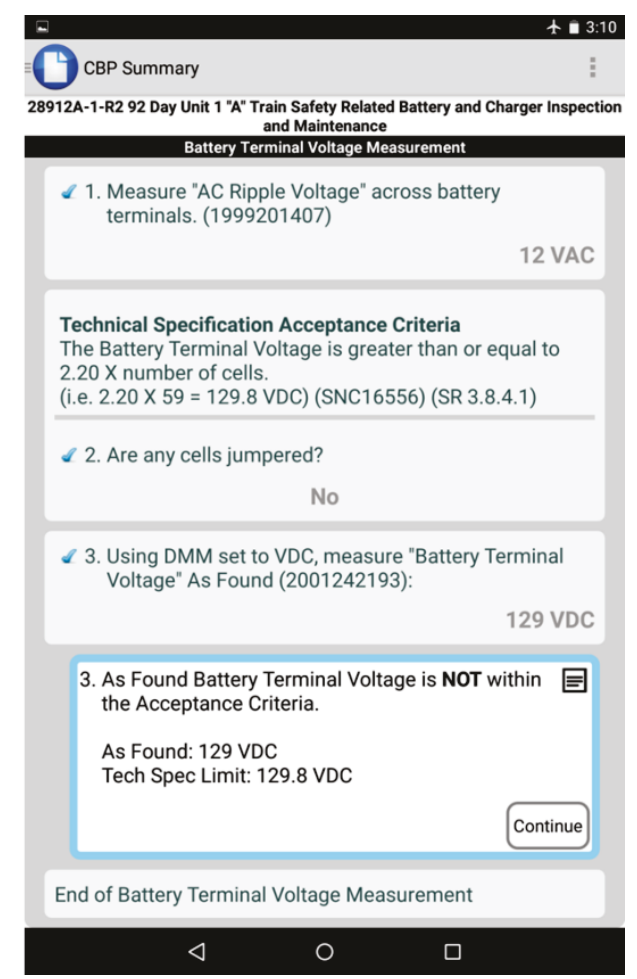

(b) Value out of range example.

Figure 20. Example of simplified step logic and recorded value out of range.

Vogtle does not use barcodes in the plant, but they were willing to temporarily add barcodes on components relevant for the battery and charger tests and inspections. Hence, the AWP system will direct the technician to conduct the CCV by scanning the barcode adjacent to the equipment identification tag. The visual cue in the AWP system is the "Scan Equipment ID" button in the procedure step, as shown in Figure 21(a). The button does not only provide the cue to scan the barcode, it also reminds the technician of which component to correctly identify and verify. The system will match the barcode with information stored in a database locally located on the mobile device. If there is a match, the digital CCV was successful and the technician can continue the step. As a visual cue for the correctly verified component the equipment identification tag is written in green text. If the digital CCV failed the equipment identification tag is written in red text, and the system will notify the technician as shown in Figure 21(b). The failed verification is also visually displayed by the equipment identification tag written in red above the scan button. The technician will get the option to either rescan the barcode or to conduct a manual verification using the human performance tool prescribed by the utility.

\subsubsection{Vogtle Field Evaluation Method}

The participants in the evaluation study include the maintenance technicians tasked to conduct the battery and inspection each week throughout the duration of the study. During the validation activity the research team provided training on the AWP system to the involved maintenance technicians. These technicians were instructed to train their peers on the AWP system. 
The duration of the study is planned to be 15 weeks long. It is expected that the study will result in data for 15 uses of the AWP system; however, it is likely that several participants will conduct the task multiple times. The first few times the task is to be performed, the technicians will be instructed to lead the task with the paper copy of the work order and follow with the AWP system. This is to validate that the AWP system follows the intent of the work order and that there are no unexpected glitches. It is expected that the technicians will conduct most of the evolutions of the task leading with the AWP system during the duration of the field evaluation study. When doing so, the technicians will bring a paper copy with them to the work site to use as a backup if needed.

A survey similar to the one used in the previous field evaluation study was developed and the participants were asked to fill it out after completing the battery and charger test and inspection task with the AWP system. The goal of the survey was to assess the usability of the AWP system and device. The survey was also developed to gain more detailed feedback on the design of the user interface and the overall experience using the computer-based work order.

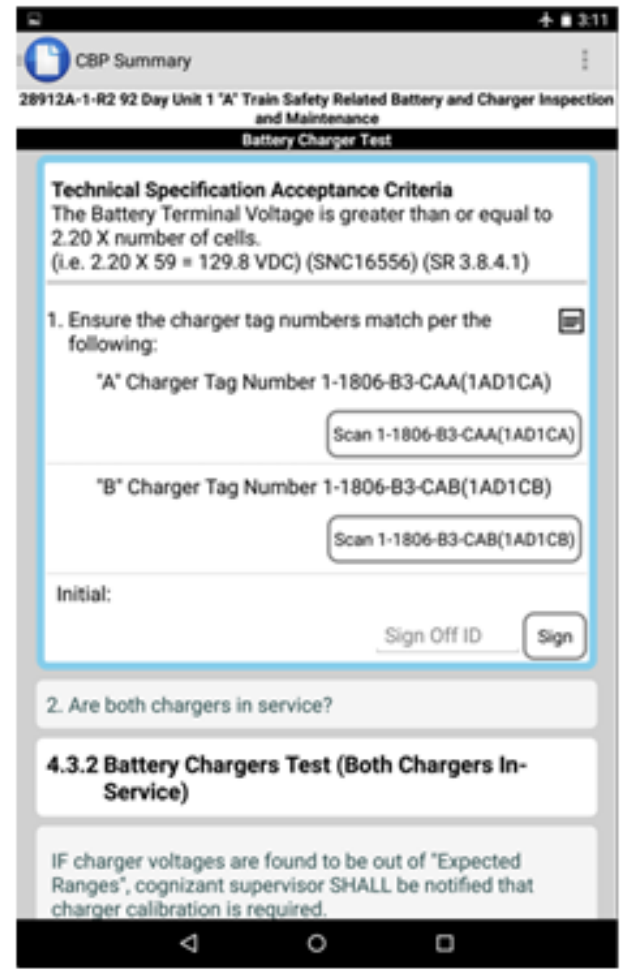

(a)

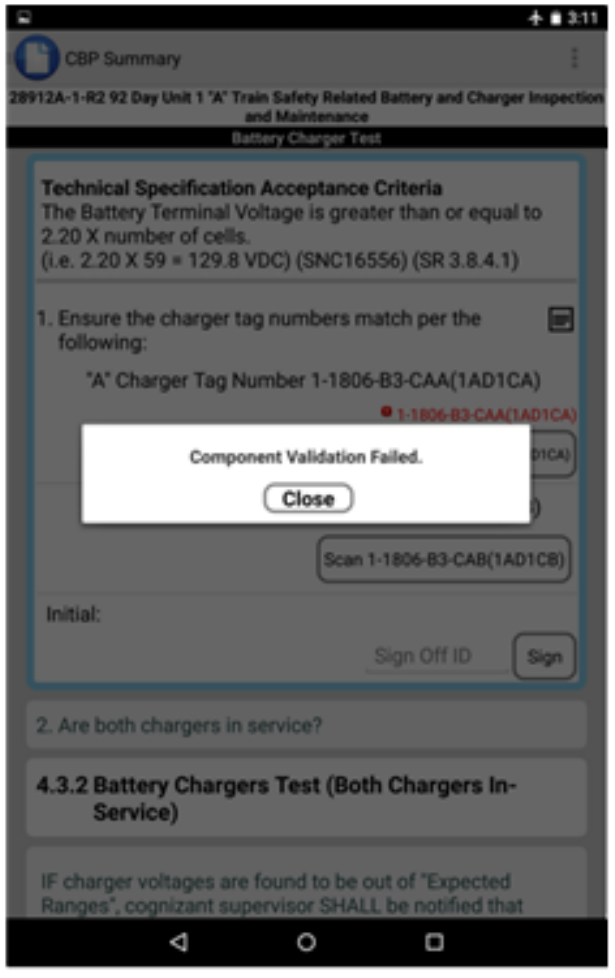

(b)

Figure 21. Example of digital correct component verification.

\subsubsection{Discussion of Initial Results}

The field evaluation study at Vogtle 1 and 2 is still ongoing and the results of the study will be documented in a later milestone report. However, some maintenance technicians have already provided feedback.

Due to a recent incident of actions mistakenly being taken on incorrect equipment the technicians welcome the option of digital CCV. In the specific work order used in the study, the technicians will use the digital CCV to ensure they are in the right unit, on the right train, and in the correct battery room or charger room.

The technicians also like how streamlined the execution of the task has become when using the AWP system compared to the paper version. The greatest efficiency gain seems to be the automated generation 
of the data sheets. When using the paper version, the technicians spend a large amount of time going between the procedure steps and the data sheets. This is no longer needed when using the AWP system. The automatic generation of the data sheets also has the potential to reduce risk of human errors commonly associated with frequent movement within a procedure, such as unintentionally missing a procedure step or conducting steps out of sequence.

Another function that has great potential for both human error reduction and efficiency gains is the way the system provides relevant information to the technician when needed. In the case a recorded value is outside the acceptable range and a conditional report needs to be filed, the AWP system directs the technician to do so as well as providing information about the acceptable range and the actual recorded value (illustrated in Figure 20).

\section{INSTRUMENTATION AND CONTROL OF ISU FLOW LOOP}

One of the activities associated with the AWP project is to develop and demonstrate means for automatic and wireless acquisition of plant process and components status information into the AWPs on a mobile device (Agarwal, Oxstrand, and Le Blanc 2014). To enable this automatic acquisition of data, it is necessary to design a platform for data exchange between the field instruments and the mobile devices. An earlier report of this project proposed an initial set of AWP requirements (Agarwal, Oxstrand and Le Blanc, 2014). This work targets the requirements associated with an architecture design that is prompt, robust, and interoperable with any technology.

The development of the automatic data acquisition aims to reveal issues and solutions generalizable to large scale implementation of a similar system. Specifically, the targeted issues and performance aspects are relevant to the communication infrastructure from the perspective of promptness, robustness, expandability and interoperability with different technologies. The objectives of the instrumentation and control scope of this work are to design a platform for a data acquisition system of wireless instruments that is expandable to an industrial scale implementation, robust to communication disturbance, interoperable with any communication technology, and that promptly reflects the process status at the prototype scale. In addition, this work will design, develop and implement an application to dynamically reflect the process status via a set of graphics. Once the system is developed, it is implemented on a laboratory-scale flow loop to evaluate and demonstrate its performance. For a successful implementation, it is necessary to demonstrate the path of communication for data exchange between a set of wireless instruments and a mobile device via a database. This enables the mobile device application to dynamically reflect the status of the flow loop instruments. The robustness and flexibility of the system is demonstrated through enforcing the loss of communication of system components, then demonstrating the effect of this loss on the system's performance. Several examples of industrial field activities are demonstrated through procedures for valve's alignment and instruments calibration.

In parallel with the activity at Vogtle the researchers developed a prototype for plant component status acquisition in a mobile device. The prototype was used to demonstrate wireless access of plant status on a mobile device. The following sections will describe this effort in great detail. The developed plant components status acquisition prototype for mobile devices will be used in future research activities in the AWP project, which aims to integrate the acquired plant performance data into the AWP system. Once this is achieved, the AWP system will be able to perform an online status verification of process alignment, thus produce self-driven procedures, which are procedures that automatically acquire the state of the plant equipment and use it to determine if the prerequisites condition for a procedure's step has been met. It will reduce the need to manually insert values into the procedures, which is expected improve the worker efficiency and reduce the probability of human error.

\subsection{Laboratory-Scale Flow Loop}

The demonstration was conducted in a laboratory-scale flow loop facility at the ISU's Energy Systems Technology and Education Center. The layout of the flow loop represents an abstract version of 
a Condensate Polisher Pool (CPP) system. The actual and graphical figures of the flow loop are shown in Figure 22 and Figure 23 The flow loop consists of nine manually operated valves, two pumps, two heat exchangers, and several pressure, temperature and flow measurement instruments. The valves used are rising stem gate valves (Valves 1, 2, 5, and 6), rising handle globe valves (Valves 3 and 4) and butterfly valves (Valves 7, 8, and 9). The flow loop has a capacity of 200 gallons per minute. It contains two flow streams referred to as the yellow and green lines. Water is injected from tanks into the flow loop through 4-inch pipes and then back into the tanks. The two streams have a cross connection and can be connected in different setups to simulate different operational scenarios. (For more details on the flow loop, see EPRI 2015).
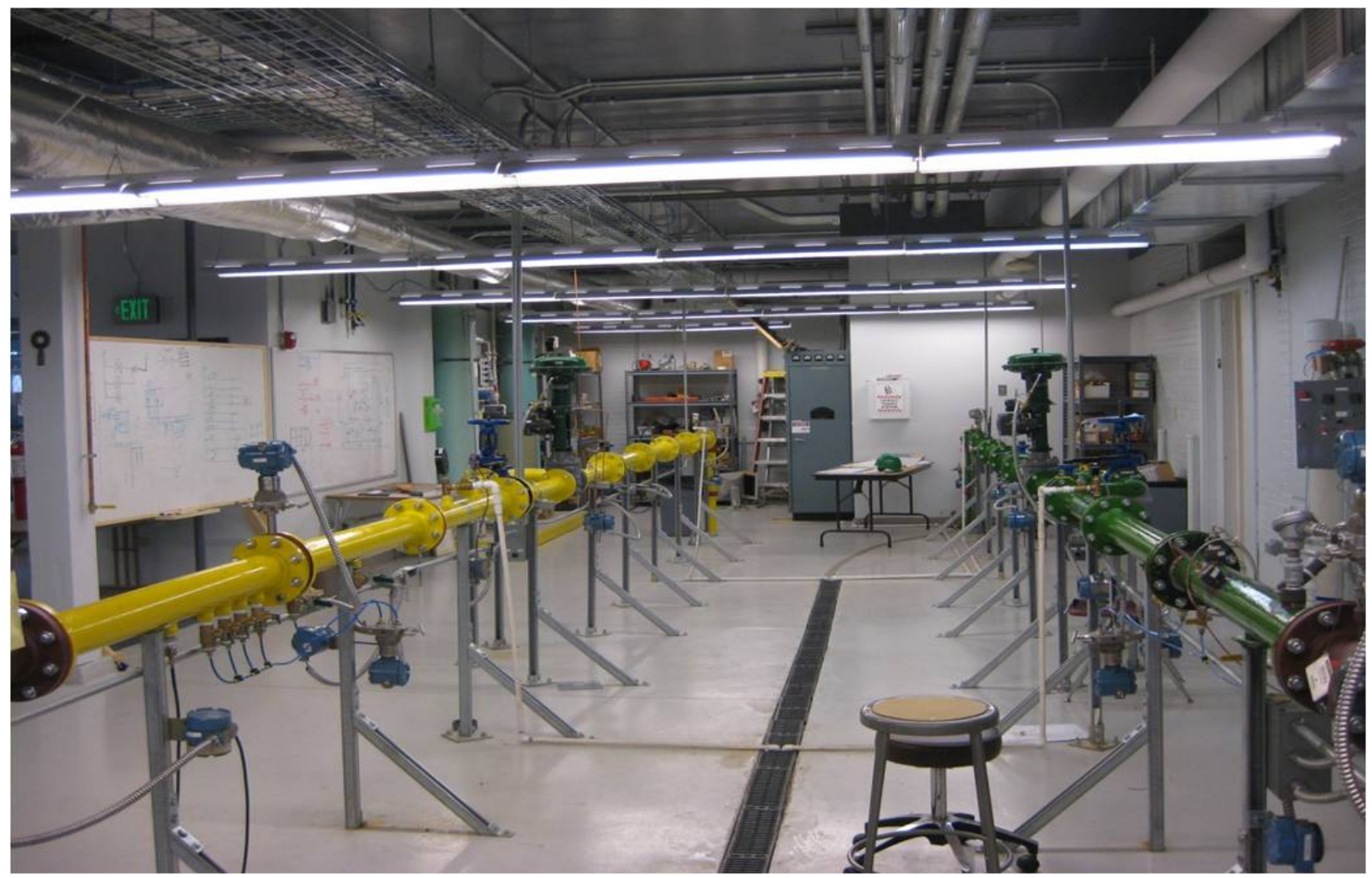

Figure 22. Picture of the laboratory-scale flow loop (EPRI 2015).

An earlier INL project (EPRI 2015) instrumented the manually operated valves with wireless position indicators. Each valve was equipped with a resistive sensor, an analog-to-digital converter (ADC), and a Wireless Fidelity (Wi-Fi) enabled Access Point (AP). The state of a valve, representing how open or closed it is, was converted to a voltage using the resistive sensor. The voltage was then captured by the ADC, encoded, and converted to ModBus packages, which is a very common communication protocol used in several industries due to its simplicity and robustness. The packages were then sent to the Wi-Fi enabled AP, which transmitted the data through a wireless network to a Programmable Logic Controller (PLC). The PLC decoded the ModBus packages and presented the status of the valves on a mobile device. The objective of that project was to develop an intelligent plant configuration management system by enabling wireless communication of the valves' position sensors. 


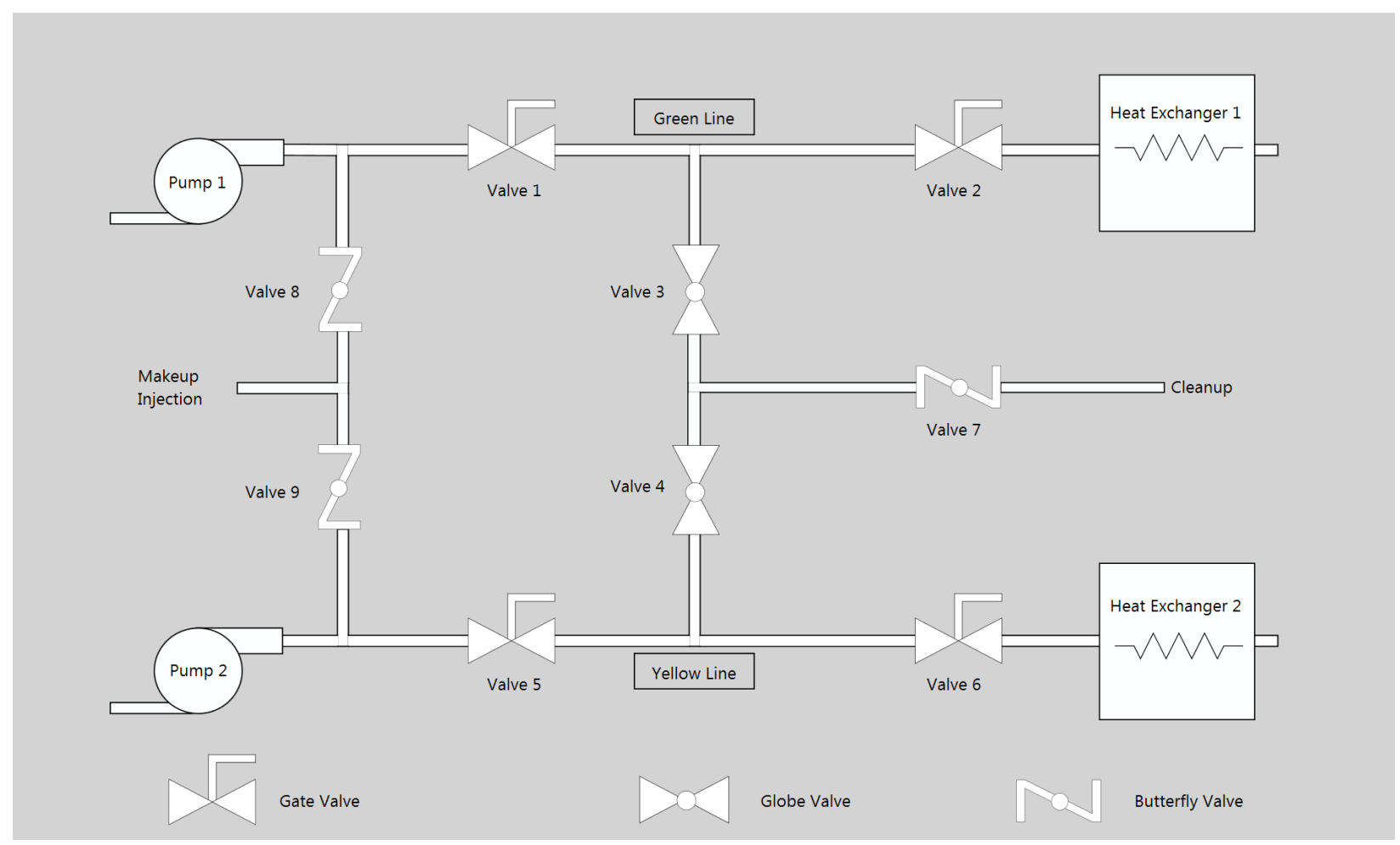

Figure 23. Graphical representation of the laboratory-scale flow loop.

\subsection{Prototype Architecture}

The architecture of the plant components status acquisition prototype consisted of a mobile device, an Structured Query Language (SQL) server to hold the prototype's database (DB), and the valves' wireless APs as wireless instruments. The tablet was connected through the SQL DB to the valves' as shown in Figure 24. The SQL server was used as a translator and a hub between the valves and the tablet. The tablet communicates through a wireless router with the SQL server only. Likewise, the valves communicate through the same wireless router with the SQL server only. As a result, the valves and the tablet do not directly communicate with each other. The paths of communication are shown by the solid and dashed double-arrows of Figure 24.

The technology selection process is application dependent and is based on several criteria (Farris and Medema, 2012). In this prototype, a Windows ${ }^{\circledR}$ Surface 2 Pro tablet was used as an example of a mobile device. The tablet was loaded with an application for the dynamic representation of the flow loop. The application was designed and developed in C\# programming language. The choice of a Windows-based device for this application was to demonstrate cross platforms capabilities, and to use earlier C\# graphical elements libraries.

An SQL DB was created with nine tags representing nine manually operated valves. A field communication application was also programmed in C\#, and was loaded into the SQL server. The field communication application is responsible for the data exchange and translation between the wireless instruments and the SQL DB. The existing Institute of Electrical and Electronics Engineers (IEEE) 802.11-based network infrastructure of the flow loop was used. 


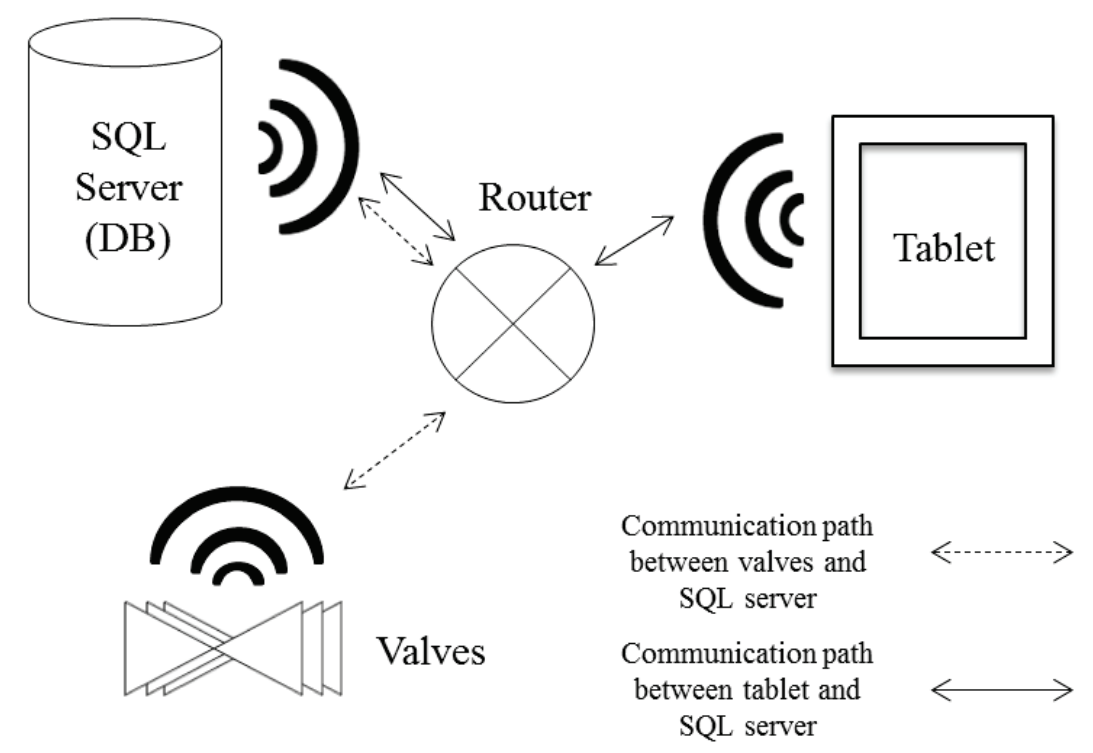

Figure 24. Wireless network architecture of the prototype.

\subsection{Mobile Device Application}

The application was researched and developed based on an object-oriented architecture. A set of static and dynamic objects, such as valves, pumps, heat exchangers, and pipes, are represented by symbols, and are used to construct Figure 25, which represents the overall view of flow loop. This graphic was developed with an approach that enables a scalable window to support any size of tablet, or any of the mobile devices listed in Farris and Medema (2012). Each of the flow loop instruments or actuators in the user interface is connected to a unique identifier, which is the tag name. The tag name is used as a common identifier for the instrument in the user interface, field instruments, and SQL DB. The SQL DB acts as a focal point for data distribution between the field and the mobile devices. This means that the mobile device application does not locally store a tag's state or value. It simply reflects the current state or value of a tag from the SQL DB. A command to change a state or value (e.g., for calibration) via the application is reflected into a request of change to the SQL server. Once the SQL server processes the change and updates the DB, the application and other applications on other mobile devices read the updated state or value from the DB. Any change of the instrument's state or value in the field is reflected in the SQL DB and in the application's user interface. This centralized concept of data update is necessary because, in an industrial scale implementation, a large number of applications on mobile devices are used, and the updated tag needs to be reflected on all devices instantaneously. The following subsections will explain the design of the dynamic symbols and faceplates.

\subsubsection{Dynamic Symbols}

A dynamic symbol is a graphic element that changes its color, text, visibility, size, orientation or any other characteristic based on a change of state or value of one or more tags. This implies that every dynamic symbol is assigned one or more tag names. In this prototype, three valve dynamic symbols were developed representing the three valve types used in the flow loop. The valves' dynamic symbols were set to change their color based on the state of the valve. The valve's symbols and their colors legend are shown in Figure 26. The used colors were chosen to sustain consistency with the flow loop PLC colors, but can be easily changed as needed. 


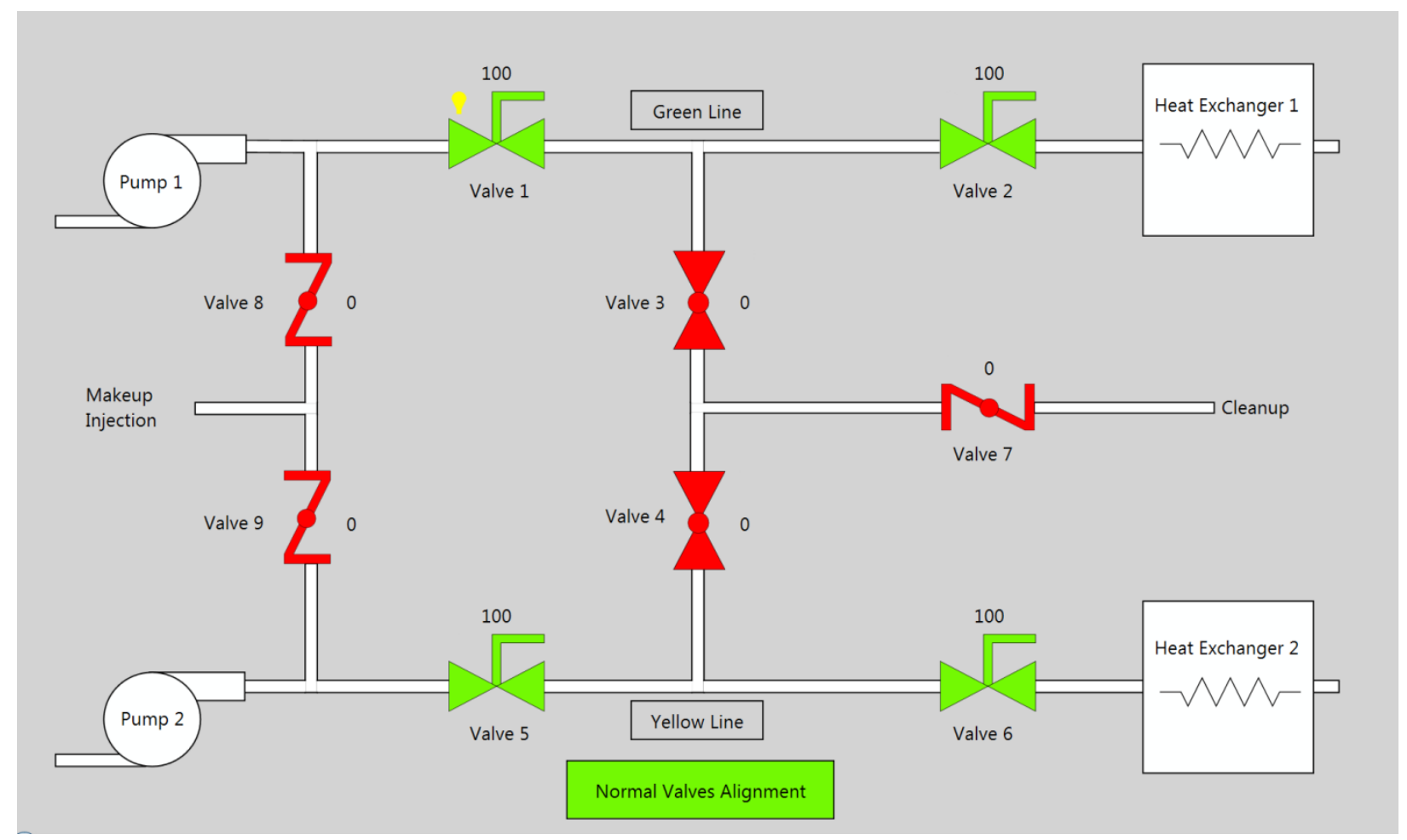

Figure 25. Main graphic of the flow loop.

To link the valve's dynamic symbols to the DB, each dynamic symbol was assigned one tag in the application and the same tag in the SQL DB. The symbol reflected one of the tag's columns, which represented the position of a valve in a scale from 0 to 100 . Due to the noisy nature of the flow loop sensors, a position definition that accounts for mechanical hysteresis was needed. A value of $0 \pm$ mechanical hysteresis was defined as a closed valve and a valve of $100 \pm$ mechanical hysteresis was defined as an open valve. The mechanical hysteresis was set as $5 \%$ in the application, but can be changed to any value depending on the type of instrument used. It is possible, as part of future work, to move the definition of mechanical hysteresis to the DB. This will enable it to be tag specific. A dynamic text symbol was developed to show the value of the valve position, which can be seen above or adjacent to the valves in Figure 25.

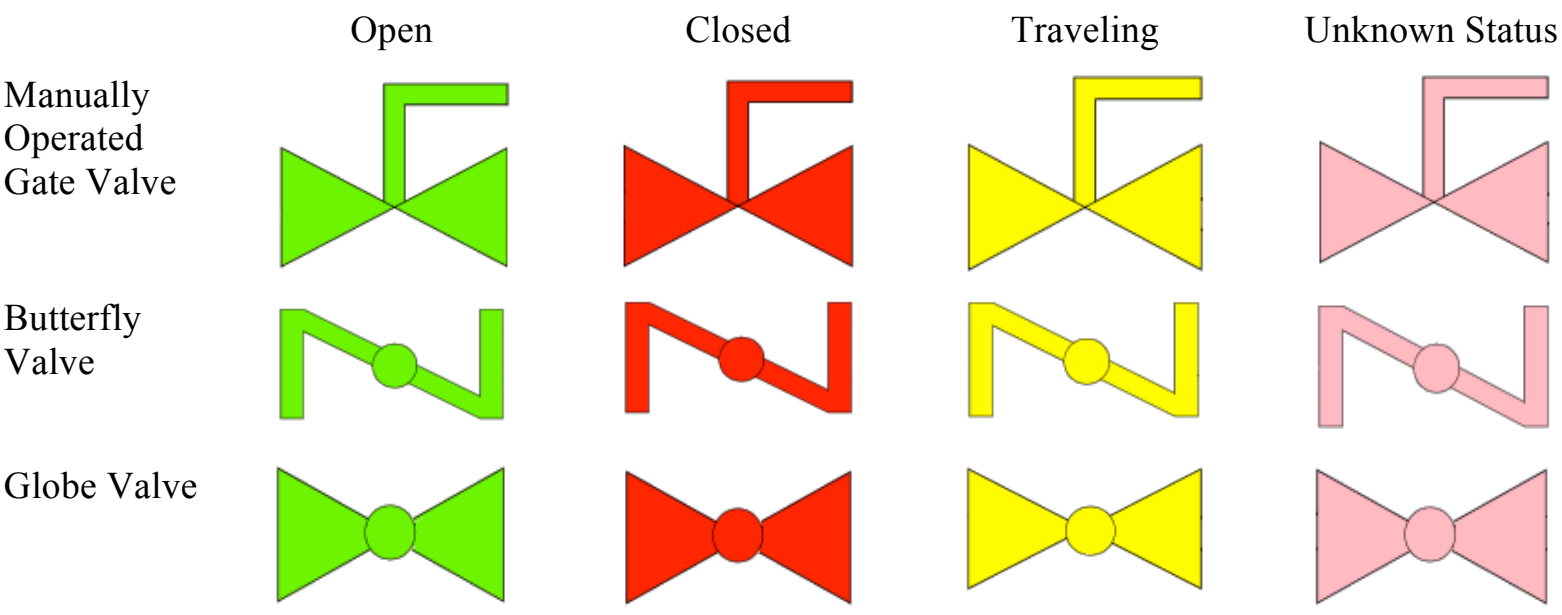

Figure 26. Symbols of valves and their dynamic color of state. 
The state of all the valves in the main flow loop graphic is read into a special dynamic symbol. This symbol was designed to determine if the valves alignment matches one of the defined scenarios in EPRI (2015). The symbol presents an indication of the readiness of the process to perform a certain procedure. This indication is an early and simple demonstration of the future plans to incorporate the automatic acquisition of the process status into procedures to determine automatically the readiness to perform a certain step. The green box at the middle bottom of Figure 25 indicates that the valves are in the normal valves alignment.

A lamp symbol was used to indicate the state of a light emitting diode (LED) at the instrument side. This is a digital tag element that is implemented to enable the field operator to turn on, from the application, an LED on the valve's ADC. This is used to verify that the work is performed on the right equipment. If the field LED is on, a lamp symbol will be apparent next to the valve in the user interface, as shown next to Valve 1 in Figure 25.

\subsubsection{Faceplates}

It is often required by plant operations that when a dynamic symbol is clicked, a faceplate should be displayed. A faceplate is a graphical element that displays one or more elements of a tag in various formats. The faceplate usually has a higher level of details than its parent graphic. The faceplate developed for a valve in this data acquisition prototype is shown in Figure 27 (a). The faceplate consists of dynamic elements that are used to show the valve's position, control the lamp, and access another faceplate for calibration. The current state of the valve is highlighted in yellow, which implies that the state of valve 1 in Figure 27(a) is open. The lamp button will toggle the state of the LED.

The calibration button in Figure 27(a) will open a new faceplate similar to the one shown in Figure 27(b). The calibration faceplate displays the current voltage value of the valve's sensor, and the voltage representing the closed and open states of the valve, which are the calibration high and low set points. The use of these set points will be explained in a later section. These values are directly read from the SQL DB. The calibration process is performed by a field operator changing the valve's position to the closed or open states, then pushing the calibration "set open" or "set closed" button that corresponds to the current field state. This will store the current voltage value as the calibration high or low in the SQL DB. Though the calibration commands are initiated by the application, the transformation of raw data to position is performed by a field communication application instead of the application. This implies that only one application will transform the raw data into a position indication instead of several transformations in each of the applications.

\subsection{Communication}

Various communication standards were used for the development of the data acquisition prototype. The nature of data exchanged, at every stage of the communication from the valves to the application on the mobile device, resulted in the data transformations shown in Figure 28. These transformations will be explained in the following subsection. They are application-specific, and were mainly due to the data encryption and the protocols used in the ADCs of the flow loop. A field communication application is assigned the translator role. Its task is to unpack the ModBus package, decode the data, and then store it in the SQL DB

In an industrial scale implementation, multiple similar or different field communication applications can be loaded on one or more servers, and possibly communicate with one or more SQL servers. If an ADC is added to the system with a new type of data encryption or communication protocol, a new field communication application would need to be developed for that specific data encryption or protocol. The field communication application is thus a modular element that is instrument-dependent. 


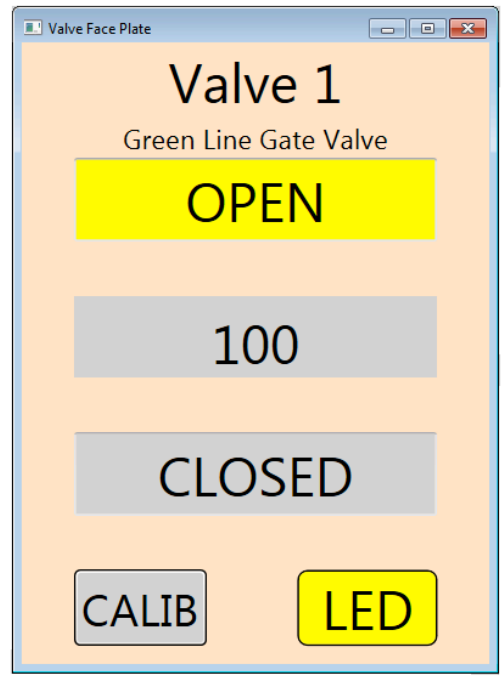

(a) Main faceplate.

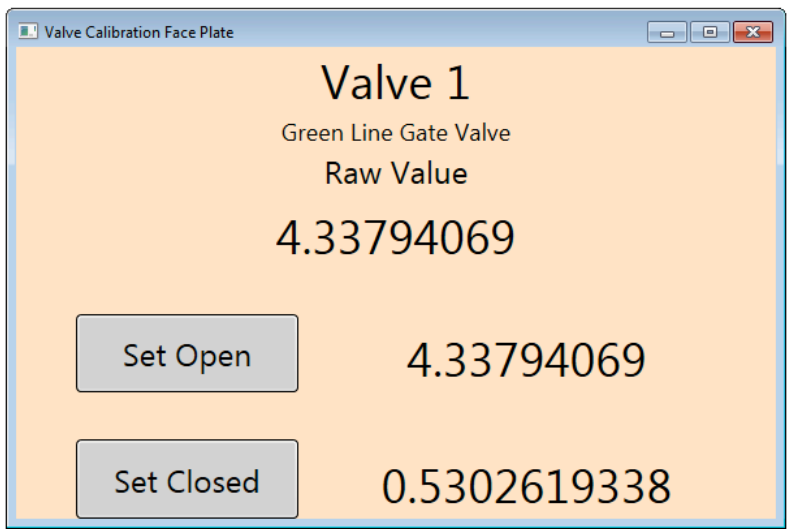

(b) Calibration faceplate.

Figure 27. Valve's faceplates and calibration faceplate.

A communication package can have multiple wrapping before it is sent, and multiple unwrapping when it is received. Each wrap is typically performed using a different communication protocol that serves specific functions. The base wrap in the used wireless network is the Transmission Control Protocol/Internet Protocol (TCP/IP). The transformations in Figure 28 are performed over the base $\mathrm{TCP} / \mathrm{IP}$. As long as the base communication is TCP/IP based, the higher communication layer can be of any higher level protocol. The interfacing in this scenario is software-based only, and no hardware interfacing module is needed. If a non-TCP/IP device is to be integrated, a dedicated interfacing module to the TCP/IP network would be needed. The following subsections will explain in more details the architecture of the field application interface with the SQL DB, and the application interface with the SQL DB.

\subsubsection{Communication between Field Instrumentation and SQL Database}

To transmit the digital data representing a float voltage value from the valve's position sensor, the ADC transforms the analog data into a digital format, which is a binary representation of the analog value. The digital data is then transformed into the IEEE Standard for Floating-Point Arithmetic (IEEE754) format. This standard enables encoding float values into binary numbers. It is based on splitting the binary bits of the digital data into three sets with different interpretations. These sets consist of one bit for the sign, a defined number of bits for a float value, and the remaining bits for an exponent. More information on the standard can be found in IEEE Standard 754 (2008). After the IEEE754 transformation, the digital data is packed into a ModBus TCP/IP packet (Schneider Automation 2006). 


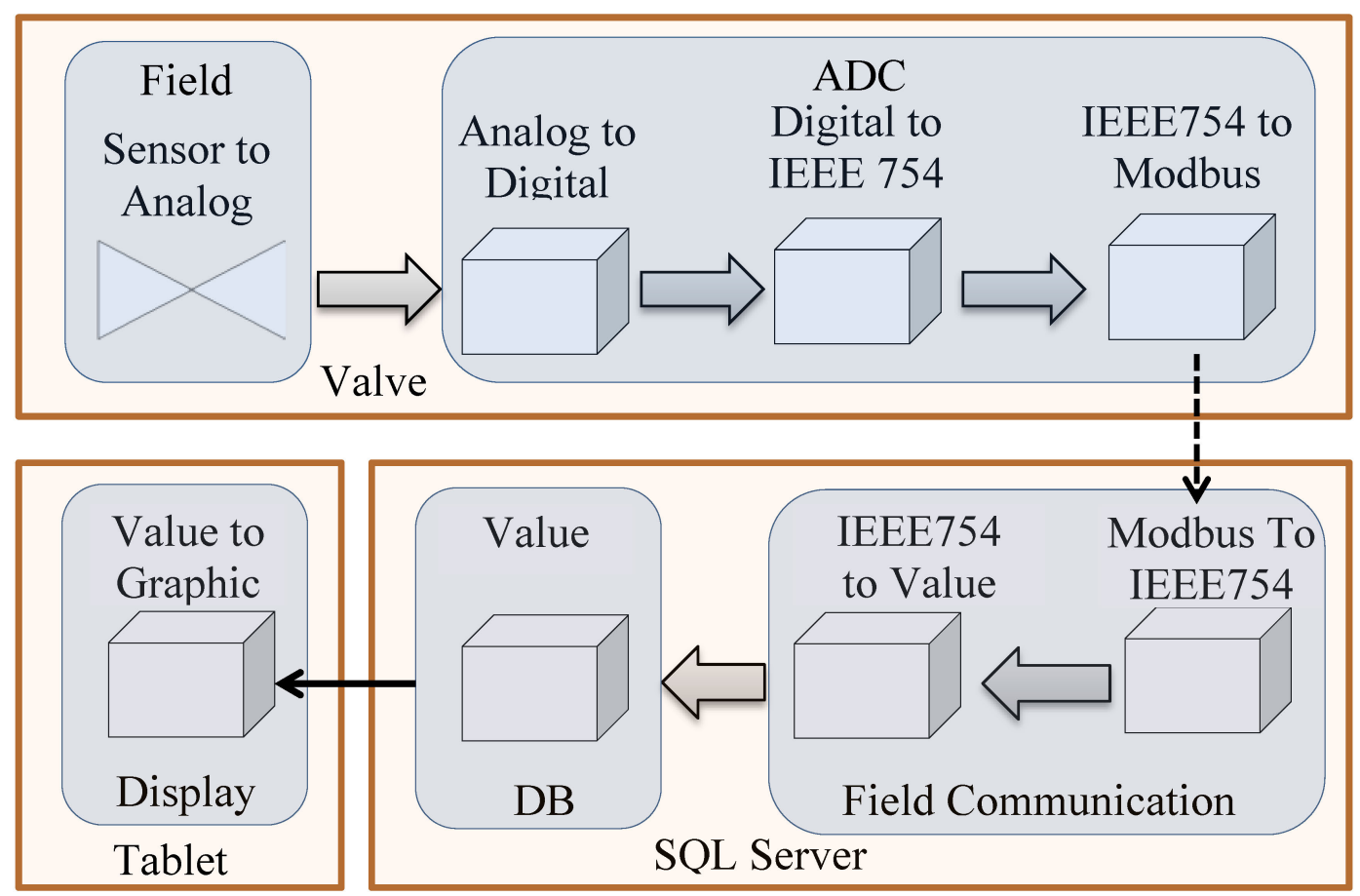

Figure 28. Data format transformation from the field to the tablet.

ModBus relies on a master and slaves architecture. The master initiates a request and the slave responds to the request with a reply or an acknowledgement message depending on the type of master request. The protocol was originally designed for a physical form of serial communication. Each message containing an address of the targeted slave is broadcasted to all slaves. Each slave examines the message to determine if the specific address matches the slave's own address. If not, the slave discards the message and waits for new messages. If there is a match (i.e., the slave is the targeted slave), it decodes the packet to determine the request then responds accordingly. A serial-based ModBus (non-TCP/IPbased) relied on a checksum to validate the packet against errors. It did not incorporate any security, since a physical link was needed. The TCP/IP-based ModBus removed the need for the checksum, since the communication packets are checked at the lower TCP/IP layer. It also benefited from the TCP/IP security infrastructure, thereby enabled the expansion of the ModBus application to wireless devices. In ModBus TCP/IP, the messages are filtered by the IP address before the slave address is read. This enabled better communication load management, and introduced a hierarchical network approach to ModBus.

In the developed prototype, the ModBus master is the field communication application. It requests to read or write data from or to slaves, which are the sensors' ADC. The ADC is accessed through an Ethernet-based wireless AP device, which is identified by an IP address. For every IP address, one ADC is assigned a slave number. Though not implemented in this prototype, it is possible to have multiple ADC slaves connected to one AP with one IP address.

For every ADC, a defined map of the analog and digital signals to ModBus holding registers and coils is loaded. This is needed as to ensure that a specific holding register or coil address has the same meaning for the ADC as it does for the field communication application. To apply a centralized data concept, the map is incorporated in the SQL DB, and is loaded into the field communication applications before they establish communication with the ADC slaves.

The field communication application implemented a timed cyclic approach. The ModBus interface of the field communication application cyclically pulled the value representing the valve's position from each ADC, and pushed the state of the LED. The frequency of the cyclic polling and pushing is 
configurable in the field communication application. If a field instrument does not respond within a configurable time, it is declared as unreachable, and the status of the instrument is indicated as unknown.

Instead of the Modbus conventional approach of sequential requests and responses with one slave at a time, a parallel approach of communication was developed. An independent thread was created in the field communication application for each slave, which resulted in an independent virtual link for each tag. An independent virtual link implies that a failure of communication in one virtual link will not affect other links, thus enhancing robustness.

The steps of one communication cycle in one virtual link are shown in Figure 29. The cycle starts by reading the ModBus map from the SQL DB as was indicated earlier. It then uses the map to communicate with wireless field instrument to capture the current position of the valve. While this is being communicated, the cycle processes five different tasks. These are to capture the current calibration high position from the $\mathrm{DB}$, calibration low position from the $\mathrm{DB}$, last known position of the valve from the $\mathrm{DB}$, last known raw data from the DB, and the current LED command from the DB. Each of these processes whether its accessing the field or the DB is performed on an independent thread and in parallel to other processes to ensure high code efficiency. The rest of data are used to calculate the new state of the valve according to Equation 1:

New position $=100 \frac{\text { Field Raw Data-Calib.Low Position }}{\text { Calib.High Position -Calib.Low Position }}$

If the new position value is significantly different from the last known position value that is acquired from the SQL DB, then an SQL update is performed. Otherwise, no action is further performed. The reason this check is implemented is to reduce the communication load on the SQL server. It ensures that only a significant change is reported to the SQL DB, and noise is ignored. The definition of a significant change is set in the field communication application. In a future effort, this can be set to each tag in the DB, especially when different types of instruments with different values are required. In this application, a $0.1 \%$ change was considered as the minimum significant change for all valve's instruments.

Every time a cycle ends, the current system time is pulled by the field communication application and set as a timestamp in the DB. This timestamp is critical in the validation of the current state of a tag in the SQL DB, which will be explained in the following section. The steps of Figure 29 are repeated cyclically for every tag until the application is terminated. Each cycle ends with a small time delay that is used to control the frequency of update. If a communication problem occurs in one virtual link, a retry is performed. The retry process continues until a defined maximum number of retry attempts is reached. Once it is reached, a longer time delay is introduced to the cyclic process, and a set state is sent to the SQL DB to indicate an outdated DB state. The maximum number of consecutive trials and the amount of time delay are defined in the field communication application. In a future effort, they can be specified to each tag in the SQL DB.

\subsubsection{Communication between Application on Mobile Device and SQL Database}

The sequence of the steps for this interface is shown in Figure 30. Every time a dynamic symbol is loaded into a display or faceplate, a certain element of the tag links the symbol to the DB. The link can be established to any element of a tag, and is a virtual independent link from all other dynamic symbols. For example, the calibration faceplate links the raw and calibration elements while the main faceplate links the valve position and LED command elements. It is possible to link multiple dynamic symbols to one tag element, and one dynamic symbol to multiple tag elements. Since the communication between the application on the mobile device and the SQL DB can be relatively slow and can affect the performance of the application response, each virtual link was performed on an independent thread. 


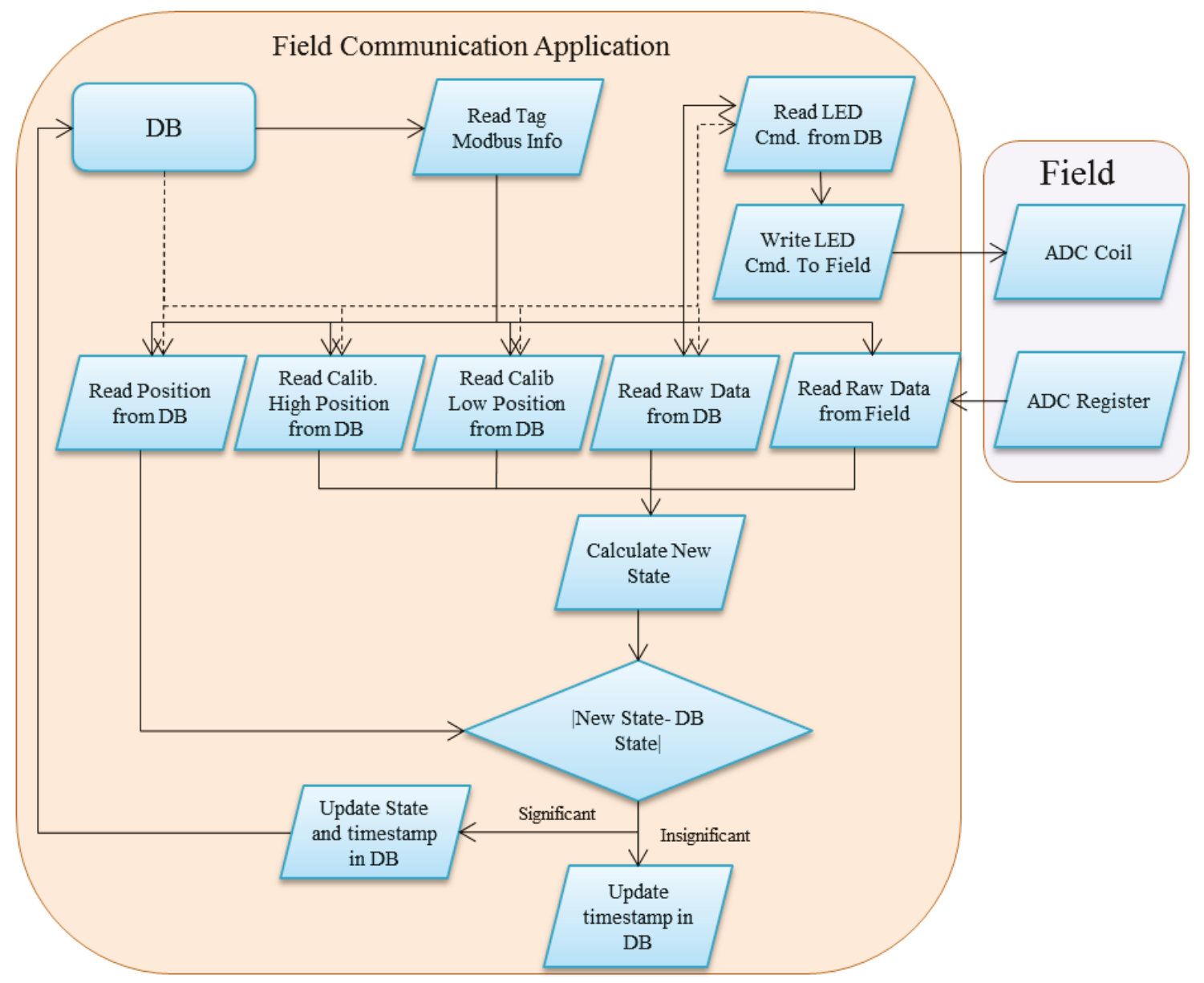

Figure 29. Sequence of steps of the field communication application.

A link can be either or both of pulling or pushing data from the SQL DB. The definition of pulling and pushing here is different than the definition introduced in the field communication application. In the field communication application, both pushing and pulling processes were cyclic. They mainly reflected whether the SQL DB is requesting data from the field or sending commands to it. In this application, a pulling mechanism is a cyclic communication process, but the pushing mechanism is not. The pulling mechanism is set for every linked tag element. The frequency of pulling data from the SQL DB is controlled by a time delay that is set in the mobile device application. As the number of open graphics increase, the communication load of the pulling mechanism increases, thus it is always advisable to optimize the frequency of pulling to be as high as possible without overloading the SQL server or the communication link. If an SQL pull request fails due to a potential connectivity issue, the frequency of pulling reduces. Whenever the connectivity is restored, the pulling frequency is automatically restored to the normal pulling frequency. The pushing mechanism is quite different. In this mechanism, the SQL server sends updates to the application as soon as an update occurs in the DB. If no DB update occurs, no updates are sent to the application. The definition of a DB update can be set at any level (i.e., it is possible to monitor multiple tags and trigger a common update notification, or to monitor certain elements of a tag and trigger a very specific update notification). It is also possible to monitor one tag and trigger an update of another when the first tag changes. Unlike the pulling mechanism, which is controlled by the application, the pushing mechanism is registered in the SQL server, and is controlled by the SQL server. A timeout can be set to this mechanism too. This enables it to behave like the pulling mechanism. 


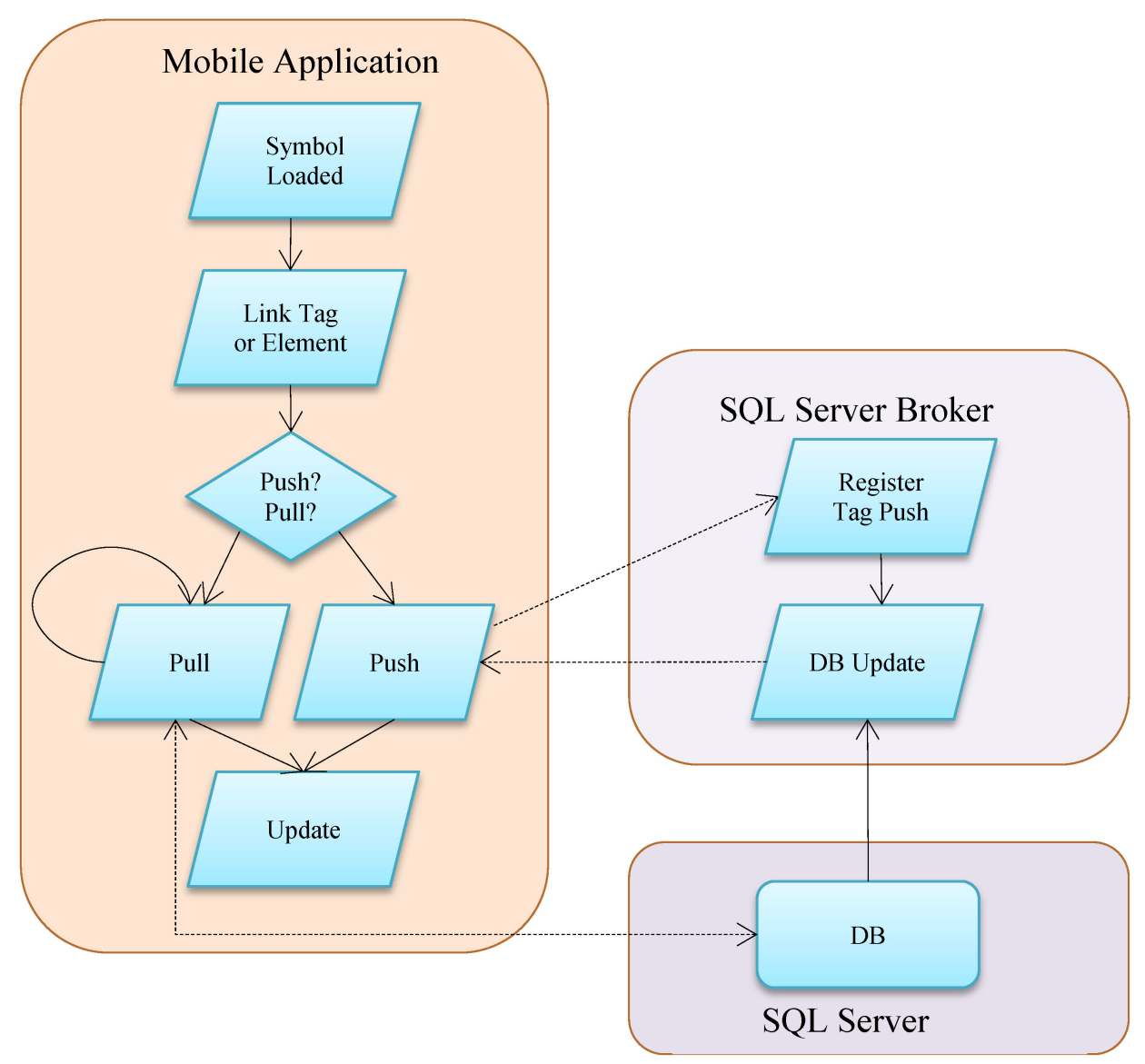

Figure 30. Sequence of steps of the mobile device application.

It is the system designer decision to choose which update mechanism is suitable. The key target is to keep the communication load as low as possible while sustaining a rapid update rate. If a certain value is continuously changing, such as a sensitive flow meter, it is logical to use a pulling mechanism for it, since a pushing mechanism will continuously trigger an update, and overload the communication link. On the other hand, if a certain value is mostly steady, a pushing mechanism is more logical. It is possible to automate the process of switching tags from the pushing and pulling mechanisms based on their historical behavior. This remains for future research.

Once data is pulled or pushed into the application, an update is performed. The update behavior is encoded in every dynamic symbol, and is specific to that symbol. This implies that the change of one tag element can cause different behaviors in different symbols. For example, the change of a tag state to $100 \pm$ mechanical stresses will change the faceplate's open box from a grey box to a yellow one, while the same change will cause the valve's color to change from yellow to green.

\subsection{Communication Performance Optimization}

To develop a platform for a large industrial scale implementation, the communication was enhanced in terms of its robustness, error handling and parallel processing. The following subsections explain the means of enhancement in these topics.

\subsubsection{Robustness and Error Handling}

To ensure robust communication between all components of the system, a persistent approach was implemented. This implies that a failure to communicate, between the application and the SQL DB or the 
field communication application and the SQL DB, resulted in a longer wait but did not drop the connection.

A communication failure of any component of the system was indicated and reported by the calling component. A communication failure in the field was detected by the field communication application. The field communication application reported the failure to the application by setting the state of the instrument to a defined outdated-data value in the SQL DB. The mobile application pulls this value from the DB as part of either the pulling or pushing mechanisms. The value is read as a normal value, but since it falls outside the range of good data, the dynamic symbols that are linked to this value will reflect a bad data status.

A failure or shutdown of the field communication application is harder to detect, because it does not update the DB to indicate its termination, and because a direct communication between the application and the field communication application does not exist. As a result, the mobile applications have no means of detecting that the field communication application was stopped. To enable the detection of a failure or shutdown of the field communication application, a timestamp was added to the DB. Every time a tag is read from the field by the field communication application, the timestamp is updated in the DB. The timestamp serves as an indication that the data is up to date. Its update occurs regardless of whether the tag was actually updated or not, as long as the field communication is healthy. Every time a tag element is acquired from the DB, the application evaluates the timestamp to determine its validity. If the timestamp shows that the data is older than a certain time threshold, set in the application, the data is declared outdated and a time out value is displayed in the application, instead of the actual received data.

If an SQL DB failure occurs, it is detected by both the field communication application and the mobile device application. The field communication application will continue to try to communicate, but at a slower rate until the communication is restored. The application on the mobile device will follow the same strategy, but in addition, it will change the dynamic symbols displays to a defined bad SQL connection value.

A failure or shutdown of the mobile device application is not detected by any component of the system, since this will probably be detected by the user. Once an application is shutdown, it removes its links from the SQL push subscription. If the application was shutdown in a manner that prevents it from safely removing the pushing mechanism subscription, the timeout of the subscription detects the loss of communication and terminates them.

A failure of any link is indicated as a failure of communication with the component. The developed applications do not distinguish between a failure that occurred at the link level or the device level. If multiple failures occur, the closes failure is indicated as the cause of failure only. For example, if a failure occurs at both of a field device and the SQL server, the bad SQL connection value will be displayed.

\subsubsection{Parallel Processing}

To improve the execution efficiency, and avoid lags in any of the system's elements, all tasks that require extensive computational resources or are relatively slow were assigned to an independent thread. This does not constitute a problem to the application, since each will have few open graphics, which corresponds to few running threads. However, this is not the case for the field communication application.

The field communication application utilizes a separate thread for every tag. Each of these threads is spanned into several threads as was shown in Figure 29. Since the number of threads that can be used is limited by the available memory and system performance, the number of simultaneous virtual tag links between the field communication application and the field instruments is limited too. To cap the number of threads that a field communication application can use, a first-in-first-out approach can be applied. This will result in partial sequential processing of the virtual links, but will enable the expansion of the application's domain to any number of tags. Partial sequential processing implies that each tag will have to wait for its turn on the threads pool before communicating with the field. The number of allowed 
threads will control how long a tag has to wait. If the number of tags is high, a command-driven approach can be implemented, or multiple servers can share the load to overcome the longer waiting time of the first-in-first-out approach. A command-driven approach implies that data are only pulled from the field when a request of update is issued from the mobile device application by the user. While this approach has its load reduction advantage, it has many limitations including a slower response and the lack of early detection of an instrument's failure. Load sharing implies a modular approach of the communication architecture, which is suitable for industrial scale implementation.

In an industrial scale implementation, several tablets running the application would be connected to a large number of instruments through one or more SQL servers. Additional SQL servers could be included to mirror or backup the SQL DB or to share the communication and server loads. These servers can be connected through a wired or wireless connection. An example of the load-sharing concept for data acquisition through two systems, one of which has redundant set of servers, is shown Figure 31. In Figure 31, the set of Instruments 1 only communicates with the set of redundant SQL Servers 1 and 2, and the set of Instruments 2 only communicates with SQL Server 3. The mobile devices can communicate with all SQL servers. This design flexibility was achieved due the modular design for the underlying communication applications. In SQL Server 3, the modular design enabled the separation of the field AP from SQL Server 1, while maintained the AP on SQL Servers 1 and 2.

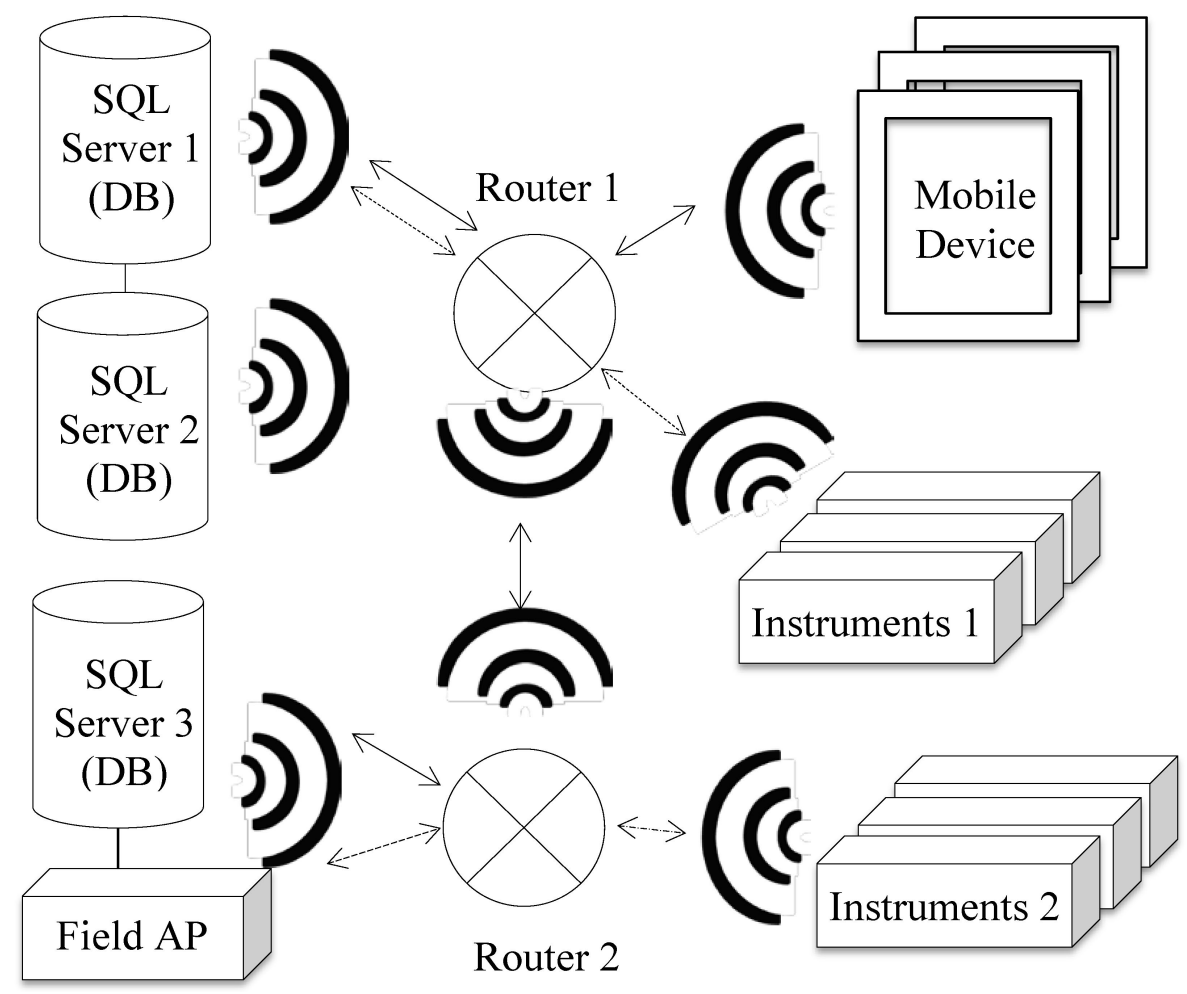

Figure 31. Load sharing wireless network architecture.

\subsection{Conclusions}

This work succeeded in developing an initial prototype for wireless data acquisition of field instruments into a mobile device through a C\# developed code at INL. As predicted in Agarwal, Oxstrand, and Le Blanc (2014), the prototype development and testing on the laboratory-scale flow loop produced various challenges, some of which were addressed in this stage, and other remain for future work as listed in the following section. 
The design criteria of focus at this stage were for the system to be to prompt, robust, expandable and interoperable with any technology. The development of a prompt system entailed significant performance optimization, including using parallel processing, parallel communication, modularity, noise filtering, and frequency tuning. A robust network was achieved by enforcing a persistent continuous data exchange that automatically detects and conveys a failure of any link or component in any part of the system, and that restores the communication once the link or component is restored. An expandable topology was enabled by modularity and enabling load sharing. The number of servers, mobile devices, and field instruments are not limited from a system structure point of view. The system interoperability was met by the independence of the field communication application. Any TCP/IP enabled field instrument can be linked to the introduced system though a customized field communication application. A non-TCP/IP field instrument can also be integrated with proper interfacing. The current system supports any type of mobile devices. The screen size of the mobile device is not restricted from a system point of view, due to the scalable nature of the user interface developed.

\section{PATH FORWARD - FY2015 AND FY2016}

In order to fully demonstrate the concepts developed in the AWP project, the team will conduct a study to show how real time status information from wireless valve position indicators can enhance the written work instructions for manual tasks. The valve position indications will be incorporated into the existing AWP system, and the team will carry out an evaluation study to investigate how to efficiently incorporate that information in the instructions used by the operator to conduct the task.

The team will use the existing valve alignment procedure for the flow loop at ISU and map out the logic to convert it into the structure needed for the AWP system. They will then integrate the information from the wireless sensors to help the operator verify that the manual task was carried out appropriately (e.g., notify the operator if a valve is in the wrong position).

The new functionality, the ability to display plant status information, will be added and demonstrated in the AWP system for the ISU Flow Loop procedures. This functionality will allow the operator to receive real-time data on any plant status items that pertains to the procedure and specifically the step the operator is currently performing. This acquisition of the plant status information can help provide feedback to the operator that an action is needed or that an action was performed correctly.

The ongoing field evaluation at Vogtle 1 and 2 will be closed out early FY16. The feedback from the maintenance technicians will be analyzed and the result will be presented in an upcoming milestone report.

In FY16, the AWP project will focus on enhancing the communication framework for wireless instruments. This framework represents the infrastructure that will enable the data exchange of the various elements of the AWP system with plant instruments. The efforts in FY15 developed the initial platform that targeted the preliminary objectives of the communication network. FY16 will expand on this effort, and investigate several topics associated with the applicability of the framework.

Different possible communication strategies will be researched and developed to allow a field operator to access information of plant components on a near real time basis. This includes developing and evaluating the capabilities for direct acquisition of instrument information, variable rate of data acquisition, load sharing, and communication prioritization.

To evaluate the developed communication framework, a virtual simulation based demonstration of tens of thousands of instruments will be performed. This simulation will highlight the bottleneck regions of the framework for further enhancement. 
In addition to enhancing the communication strategies, the communication architecture will be developed to maximize the wireless bandwidth utilization. To achieve this, a multi hop communication concept will be developed and tested. Its performance metrics like reliability, accuracy, latency, and throughput of wireless communication will be evaluated.

After the communication strategies and architecture are developed, the robustness of wireless communication to external factors will be evaluated. The external factor evaluated in this scope will be Radio Frequency (RF) interference. Noise will be generated through an external source to simulate the RF environment in a nuclear power plant, and the performance of the system will be monitored and evaluated.

The expandability of the developed data acquisition platform of wireless instruments will also be evaluated in FY16. In addition to valves position indicators, other in plant surveillance activities will be acquired through wireless remote monitoring. This will demonstrate the interoperability of the developed system with different type of sensor technologies.

A future evaluation of the expandability of the platform will target its interoperability with different wireless hardware technologies. These technologies offer solutions to challenges that face certain scenarios of implementation of the IEEE 802.1 wireless communication, such as lower power consumption.

The result of the communication framework research efforts will produce a system that is optimized and robust on the protocol and hardware level, and that is expandable to include various wireless technologies and wireless instruments. Once the communication framework is well established, the researchers will identify the remaining technical gaps and develop a plan for unattended concerns, solutions and requirements of a large scale implementation of the communication infrastructure.

\section{SUMMARY OF ACHIEVEMENTS}

In the AWP project the researcher expanded the concepts developed for the CBP system to include work orders, associated processes, and instructions. The AWP system was first designed, developed, and evaluated at the PVNGS on their HVAC preventative work order. As part of the HVAC evaluation using the AWP system, new functionalities like switch users, automatic population of values from previous $\operatorname{logs}$, and automatic manual arithmetic calculations were implemented. These functionalities enabled maintenance technicians to keep track of a single work order executed over multiple days by multiple users. The switch users option maintained a record of technician identity that conducted individual steps of the work order along with date and time. The AWP system automatically populated recorded values in future steps where the values are relevant. The system also dynamically updated the path through the work order depending on decisions made by the technician and values recorded. This functionality minimizes human errors that could occur due to incorrect manual entries of values as well as the risk of omitting steps. Calculation errors or mistakes, selection of incorrect equation, or making rounding errors while executing work order are mistakes associated with the paper process. By using an AWP system these types of mistakes are eliminated. In addition, an option was built in the AWP system that allowed technicians to review the calculation that lead to the automatic calculated value.

Following initial design, development, and evaluation of the AWP system at the PVNGS, a field evaluation study at Vogtle Units 1 and 2 was performed. For Vogtle plant evaluation, "battery and charger test and inspection maintenance," work order was evaluated. The focus was on means to streamline and improve the process of filing and archiving the completed task. To achieve this objective, two additional functionalities were added and the overall interface of the AWP system was improved. For Vogtle plant evaluation, two different mobile devices were used: Samsung 10-in. tablet and Nexus 7-in. tablet.

The menu of the AWP system was modified to allow technicians to navigate to a specific section of the work order. An additional improvement made to the AWP system before launching the Vogtle field evaluation study was to improve the user experience of recording values. Two features made a noticeable 
difference on the user experience and efficiency: automatically moving to the next text field and reduced amount of text to record. These functionalities saved significant technician time because, in paper-based work orders, a large amount of time is spent going between the procedure and the attached data sheets.

Another salient aspect of the AWP architecture is to develop and demonstrate a platform for automatic and wireless acquisition of plant process and components status information into the AWP system. To evaluate this automatic acquisition of data, a prototype was designed, developed, and evaluated on a laboratory-scale flow loop. The developed prototype functions as a preparation step for a future phase of the project that will integrate the acquired plant performance data into the AWP. At this stage, the automatic data acquisition efforts were focused towards the performance of the communication infrastructure from the perspective of promptness, robustness, expandability and interoperability with different technologies. This work resulted in significant insight on the feasibility for utilities and vendors to develop a system that achieves these requirements.

The developed prototype was demonstrated by the acquisition of valve position indications to a mobile device. The prototype architecture consisted of a tablet as the mobile device, an SQL server to hold the prototype's DB, and the valves' wireless APs as wireless instruments. The tablet was connected through the SQL DB to the valves. The SQL server was used as a translator and a hub between the valves and the tablet. The tablet communicated through a wireless router with the SQL server only. Likewise, the valves communicated through the same wireless router with the SQL server only. As a result, the valves and the tablet did not directly communicate with each other.

In the automatic data acquisition prototype, a Windows ${ }^{\circledR}$ Surface 2 Pro tablet was used as an example of a mobile device. The tablet was loaded with a mobile device application for the dynamic representation of the flow loop. This application was designed and developed in C\# programming language. An SQL DB was created with nine tags representing nine manually operated valves. A field communication application was also designed and developed in C\#, and loaded into the SQL server. The field communication application was responsible for the data exchange and translation between the wireless instruments and the SQL DB. The existing IEEE 802.11 based network infrastructure of the flow loop was used.

The communication platform was enhanced in terms of its robustness, error handling and parallel processing. To ensure robust communication between all components of the system, a persistent approach was implemented. This implies that a failure to communicate, between the mobile device application and the SQL DB or the field communication application and the SQL DB, resulted in a longer wait but did not drop the connection. To improve the execution efficiency and avoid lags in any of the system's elements, all tasks that require extensive computational resources or are relatively slow were assigned to an independent thread. 


\section{REFERENCES}

Agarwal, V., J. Oxstrand, and K. Le Blanc, 2014, Automated Work Packages: An - Initial Set of Human Factors and Instrumentation and Control Requirements, INL/EXT-1433172, Rev. 0, September 2014.

Farris, R. K., H. Medema, 2012, Guidance for Deployment of Mobile Technologies for Nuclear Power Plant Field Workers, INL/EXT-12-27094, September 2012.

Hallbert, B. P., K. D. Thomas, 2014, Advanced Instrumentation, Information, and Control System Technologies -Technical Program Plan for 2014, INL/EXT-13-28055, Rev 3, September 2014.

Hashemian, H. M., C. J. Kiger, G. W. Morton, B. D. Shumaker, 2011, "Wireless sensor applications in nuclear power plants," Nuclear Technology, Vol. 173, No. 1, pp. 8-16.

IEEE Computer Society, 2008, "IEEE Standard for Floating-Point Arithmetic," IEEE Std. 754.

Le Blanc, K., J. Oxstrand, and A. Bly, 2015, "Computer-Based Procedures For Nuclear Power Plant Field Workers: Design Implications Based On Three Evaluation Studies," Proceedings of the 9th Nuclear Plant Instrumentation, Control \& Human-Machine Interface Technologies (NPIC\&HMIT) topical meeting of the American Nuclear Society, Charlotte, North Carolina, February 23-26, 2015.

Oxstrand, J., K. Le Blanc, and A. Bly, 2015, "The Next Step in Deployment of Computer Based Procedures For Field Workers: Insights And Results From Field Evaluations at Nuclear Power Plants," Proceedings of the 9th Nuclear Plant Instrumentation, Control \& Human-Machine Interface Technologies (NPIC\&HMIT) topical meeting of the American Nuclear Society, Charlotte, North Carolina, February 23-26, 2015.

Oxstrand, J. and K. Le Blanc, 2015, Computer-Based Procedures for Field Workers Identified Benefits, INL/EXT-14-33212, Rev. 1, January 2015.

Oxstrand, J., K. Le Blanc, and A. Bly, 2014, Computer-Based Procedures for Field Activities: Results from Three Evaluations at Nuclear Power Plants, INL/EXT-1433011, Rev. 0, September 2014.

Schneider Automation, 2006, MODBUS Messaging on TCP/IP Implementation Guide V1.0b, MODBUS Organization, last accessed June 30, 2015, http://www.MODBUS.org/specs.php.

Thomas, K. and S. Lawrie, 2015, Pilot Project Technology Business Case: Mobile Work Packages, INL/EXT-15-35327, May 2015.

EPRI, 2014a, Nuclear Maintenance Applications Center: Applying Skill of the Craft to Maintenance Planning, Electrical Power Research Institute, Palo Alto, California, 3002003194, September 5, 2014.

EPRI, 2014b, Improving the Execution and Productivity of Maintenance with Electronic Work Packages: A Mobile Work Management Initiative, 3002003043, October 2014.

EPRI, 2015, Intelligent Plant Configuration Using Wireless Sensors: Application to Nuclear Power Plant Valves, EPRI, Palo Alto, Califoria, 3002005325, 2015. 